\title{
THE TWO-SIDED STEFAN PROBLEM WITH A SPATIALLY DEPENDENT LATENT HEAT
}

\author{
TERRY R. MCCONNELL
}

Dedicated to Professor Guy Johnson on the occasion of his retirement

\begin{abstract}
We prove existence and uniqueness of solutions to a problem which generalizes the two-sided Stefan problem. The initial temperature distribution and variable latent heat may be given by positive measures rather than point functions, and the free boundaries which result are essentially arbitrary increasing functions which need not exhibit any degree of smoothness in general. Nevertheless, the solutions are "classical" in the sense that all derivatives and boundary values have the classical interpretation. We also study connections with the Skorohod embedding problem of probability theory and with a general class of optimal stopping problems.
\end{abstract}

\section{INTRODUCTION}

The two-sided Stefan problem is a free boundary problem for the heat equation which models the melting of ice and other materials in one dimension. We suppose that at time zero a nonzero temperature distribution is specified in some interval and that the solid phase of the material occupies the exterior of this interval. We also suppose that the temperature of the solid phase is zero, which is also the melting point. Over time, the boundary between melted and solid material will move as more of the material melts, and the initial temperature distribution will evolve as a solution of the heat equation in the melted region with zero boundary values. The mathematical problem is to describe the melted region in space-time and the temperature distribution for all time.

The main goal of this paper is to prove an existence and uniqueness result in this context, where we additionally allow the latent heat of the material to depend on position. Moreover, both the latent heat and the initial temperature distribution may be given as positive measures rather than point functions. While we are not aware of any physical problems which require such generality, the resulting problem is quite natural from a mathematical point of view.

Received by the editors August 10, 1988 and, in revised form, July 20, 1989.

1980 Mathematics Subject Classification (1985 Revision). Primary 35R35; Secondary 60G40, $60 \mathrm{~J} 45,60 \mathrm{~J} 65,35 \mathrm{~K} 05$.

Key words and phrases. Stefan problem, free boundary problems, Skorohod embedding, optimal stopping, Brownian motion, parabolic potential theory.

Supported in part by NSF Grant DMS 87-00802 and the Alfred P. Sloan Foundation. 
The reasons for this are threefold-first, as we shall show below, the resulting problem is equivalent to the Skorohod embedding problem, an important problem in probability theory. Second, we shall see that any pair of strictly monotone functions may arise as the moving boundaries. If the initial distribution is a fixed probability measure, then there is a one-to-one correspondence between pairs of such functions and latent heat measures. Finally, the problem is equivalent to an interesting family of optimal stopping problems for Brownian motion. Some of these connections are known but, in our opinion, have never been fully investigated.

One consequence of this level of generality is that the usual methods for obtaining existence and uniqueness results in parabolic free boundary problems must be abandoned. In particular, one may not use a priori estimates of the derivatives of solutions of the heat equation at the boundary, since the boundaries may not be assumed to have any smoothness properties. The methods we rely upon are potential theoretic, and are based upon the work of H. Rost [19], P. Meyer [16], P. Chacon [6], and others.

To describe our results in detail, let $b_{-}$and $b_{+}$be given positive constants and $\varphi$ a nonnegative function supported in the interval $\left[-b_{-}, b_{+}\right]$. Suppose there is also given a nonnegative function $h$ which vanishes on $\left(-b_{-}, b_{+}\right)$. The Stefan problem, with latent heat $h$ and initial temperature distribution $\varphi$, is to find a pair of nondecreasing functions $s_{-}$and $s_{+}$on $[0, \infty)$ and a continuous function $u(x, t)$ on $\mathbb{R} \times(0, \infty)$ such that the following hold:

$$
\begin{gathered}
\frac{1}{2} \frac{\partial^{2}}{\partial x^{2}} u(x, t)=\frac{\partial}{\partial t} u(x, t), \quad-s_{-}(t)<x<s_{+}(t), 0<t, \\
u\left( \pm s_{ \pm}(t), t\right)=0, \quad 0<t<\infty, \\
u(x, 0)=\varphi(x), \quad-b_{-}<x<b_{+},
\end{gathered}
$$

and

$$
\frac{1}{2} \frac{\partial u}{\partial x}\left( \pm s_{ \pm}(t), t\right)= \pm h\left( \pm s_{ \pm}(t)\right) \frac{d s_{ \pm}}{d t}(t), \quad 0<t<\infty .
$$

We will not provide extensive references to the literature on the Stefan problem. Instead we refer the reader to the bibliography of [5].

It is not immediately clear how to generalize condition (1.4) to the case in which the function $h$ is replaced by a measure $\mu$. However, it is well known in the classical one-sided case, where one of the boundaries is replaced by a preassigned constant function, that condition (1.4) may be replaced by an integral equation. Similarly, it is not difficult to show, with the aid of Green's formula, that in the two-sided problem condition (1.4) is equivalent to the following family of integral equations:

$$
\begin{aligned}
\int_{I(t)}=\left[-s_{-}(t), s_{+}(t)\right] & (x+\beta) u(x, t) d x \\
& =\int_{I(0)}(x+\beta) \varphi(x) d x-\int_{I(t)}(x+\beta) h(x) d x, \quad 0<t<\infty,
\end{aligned}
$$


where $\beta$ ranges over the real numbers. (Of course, these are equivalent to a pair of integral equations.) These equations continue to make sense when $\varphi(x) d x$ and $h(x) d x$ are replaced by measures $\nu$ and $\mu$.

We may now state our main results.

Theorem 1.1. Let positive numbers $b_{ \pm}$, and positive Borel measures $\mu$ and $\nu$ be given, where $\nu$ is finite and supported in $\left[-b_{-}, b_{+}\right]$with $\nu\left(\left\{ \pm b_{ \pm}\right\}\right)=0$, and $\mu$ is $\sigma$-finite with $\mu\left(\left[-b_{-}, b_{+}\right]\right)=0$. Suppose also that $\mu$ has a continuous distribution function, i.e.,

$$
\mu(\{x\})=0, \quad-\infty<x<\infty .
$$

Then there exists a triple $\left(u, s_{-}, s_{+}\right)$consisting of strictly increasing (where finite) left continuous extended-real-valued functions $s_{ \pm}$and a function $u$ which is continuous on $\mathbb{R} \times(0, \infty)$, such that (1.1) and (1.2) hold. Furthermore, we have

$$
u(x, t) \rightarrow \nu \text { in } C_{b}^{*}(\mathbb{R}) \text { as } t \rightarrow 0,
$$

i.e., for any bounded continuous function $\psi$

$$
\int_{\mathbb{R}} \psi(x) u(x, t) d x \rightarrow \int_{\mathbb{R}} \psi(x) \nu(d x) \text { as } t \rightarrow 0 .
$$

Also, the family of integral equations

$$
\begin{aligned}
& \int_{I=\left[-s_{-}(t), s_{+}(t)\right]}(x+\beta) u(x, t) d x \\
& \quad=\int_{-b_{-}}^{b_{+}}(x+\beta) \nu(d x)-\int_{I}(x+\beta) \mu(d x), \quad 0<t<\infty, \beta \in \mathbb{R},
\end{aligned}
$$

hold, and the functions $s_{ \pm}$satisfy $b_{ \pm} \leq s_{ \pm}(0+)$. (Existence of the integrals here is part of the assertion. If for some $T<\infty$ we have $\lim _{t \rightarrow T} s_{+}(t) \wedge s_{-}(t)=\infty$ then for $t \geq T$ the second integral on the right-hand side is to be interpreted in the improper sense as $\lim _{t \rightarrow T} \int_{I}(x+\beta) \mu(d x)$.)

We prove the corresponding uniqueness result under a boundedness condition. (We are not certain that this is necessary in the presence of the other assumptions.) There is also a trivial condition involving the (arbitrary) choice of the initial positions of the free boundaries.

Theorem 1.2. Let $\mu$ and $\nu$ be as in Theorem 1.1. Then there exists at most one triple $\left(u, s_{-}, s_{+}\right)$such that $s_{ \pm}$are nondecreasing left-continuous extended real-valued functions on $(0, \infty)$ which are right continuous at 0 , and $u$ is continuous on $\mathbb{R} \times(0, \infty)$, vanishes for $x \notin\left(-s_{-}(t), s_{+}(t)\right), t>0$, satisfies the Tychonov bound

$$
u(x, t) \leq C e^{c x^{2}}, \quad|x|>B,
$$

for each $B>b_{ \pm}$and constants $c$ and $C$ depending only on $B$, and (1.1), (1.6) and (1.7) all hold. 
Theorem 1.1 may be deduced with some relatively routine additional work from the work of P. Chacon and others mentioned above. However, we believe it is worthwhile to present an independent proof for several reasons. First, as far as we know, the result of Chacon has never been published outside of his Ph.D. dissertation. Second, his methods rely on potential theory of general Markov processes and on the deep work of Rost and Meyer on the continuoustime filling scheme, and so we feel they are not very accessible to many analysts who are interested in the Stefan problem. On the other hand, the proof we give makes no use of the filling scheme and is elementary insofar as it relies only on classical potential theory. Finally, the proof we give has some interesting consequences in optimal stopping theory. (See $\S 5$.)

Throughout the rest of this paper we shall denote by $L$ the operator $\frac{1}{2} \partial^{2} / \partial x^{2}$ $-\partial / \partial t$ and by $L^{*}$ the operator $\frac{1}{2} \partial^{2} / \partial x^{2}+\partial / \partial t$. Also, $\Phi_{t}(x)$ denotes the function $e^{-x^{2} / 2 t} /(2 \pi t)^{1 / 2}$. This function is the fundamental solution of the heat equation in the sense that $L \Phi_{t}(x)=0$ for $t>0$, and $\Phi_{t} \rightarrow \delta_{0}$ as $t \rightarrow 0$ in $C_{b}^{*}(\mathbb{R})$.

We shall use standard notations and results from parabolic potential theory and its probabilistic counterpart which may all be found in Doob's monograph [8]. The properties of co-space-time Brownian motion, $(B(t), t)$, where $B(t)$ is standard Brownian motion, will enter into the proof of Theorem 1.2 in an essential way. We also use the time reversal, $(B(T-t), T-t), 0<t<T$, called space-time Brownian motion. We recall in particular that, if $D$ is a domain in $\mathbb{R} \times(0, \infty)$, then $G_{D}(x, t ; y, s)$, the parabolic Green function of $D$, is also the transition density of co-space-time Brownian motion killed at its first exit from $D$. (We will drop the subscript $D$ when $D$ is the entire half-space $\mathbb{R} \times(0, \infty)$.) In probabilistic arguments we shall adhere to the standard notation of the theory of Markov processes. For background on Markov processes, the reader may consult [10].

The body of this paper is divided into 7 parts. In $\S 2$ we prove Theorem 1.1 under the additional hypothesis that $\mu$ and $\nu$ have disjoint supports. The extension to the general case, which we give in $\S 4$, uses the uniqueness theorem, Theorem 1.2, and its proof (given in $\S 3$.)

In $\S 5$ we present two applications. First, we give a new proof of the solution of the Skorohod embedding problem for Brownian motion due to P. Chacon. Our analytic results effectively replace Chacon's use of the filling scheme. Secondly, we show that the existence of solutions to a rather large class of optimal stopping problems for Brownian motion is equivalent to the result of Theorem 1.1.

In $\S 6$ we collect some results concerning the influence of the data, $\mu$ and $\nu$, on properties of the free boundaries, which follow easily from our results and from other known results.

Finally, in $\S 7$ we frame some open questions and suggestions for future research.

In addition to the body of the paper, we attach a brief appendix which contains the statements of some results in which $\mu$ and $\nu$ are assumed to have 
continuous density functions, and the resulting free boundaries are $C^{1}$. These results are used only in $\S 6$. We do not give proofs since the reader may supply them by using as outlines the proofs of similar results in [5].

The author would like to thank R. Blumenthal, J. R. Cannon, A. Mandelbaum, E. Perkins and A. Schatz for informative correspondence or conversation on the subject of this paper, and the referee for numerous suggestions which have improved the exposition.

\section{EXISTENCE (CASE OF DISJOINT SUPPORTS)}

In this section we prove Theorem 1.1 under an additional hypothesis which we impose throughout this section:

$\mu$ and $\nu$ have disjoint supports.

Also, we may and do assume that $\nu$ is a probability measure. Furthermore, we shall assume that $\mu$ is finite. The case of infinite $\mu$ may be deduced from the case of finite $\mu$ by means of a limiting argument. This involves the use of a monotonicity theorem (see Lemma 4.1 below.) Details are left to the reader.

We impose no further assumptions on $\mu$ and $\nu$ until after (2.24) below.

Let $\mu$ be a finite positive measure on $\mathbb{R}$ and let $\underline{\mu}=\mu \otimes \lambda$ on $\mathbb{R} \times \mathbb{R}_{+}$, where $\lambda$ denotes Lebesgue measure. Similarly, define $\underline{\nu}=\nu \otimes \lambda$. Throughout this section the reader should beware of the distinction between $\mu$ and $\mu$ (underscored). We shall be concerned with properties of the heat potential

$$
G \underline{\mu}(x, t)=\int_{0}^{t} \int_{\mathbb{R}} G(x, t ; \eta, s) \mu(d \eta) d s, \quad t>0 .
$$

It is convenient to define $G \mu=0$ when $t=0$. The following result summarizes some elementary properties of $G \underline{\mu}$ that we shall need.

Lemma 2.1. Let $\mu$ be a finite measure. Then

$$
\text { G } \underline{\mu} \text { is continuous on } \mathbb{R} \times \mathbb{R}_{+} \text {, }
$$

$$
\partial G \underline{\mu} / \partial t \text { exists and is continuous on } \mathbb{R} \times(0, \infty) \text {, }
$$

and

$$
\partial G \underline{\mu} / \partial t(\cdot, s) \rightarrow \mu \text { as } s \rightarrow 0 \text { in } C_{b}^{*}(\mathbb{R}) .
$$

Recall that $\Phi_{\varepsilon}(x-y)=G(y, \varepsilon ; x, 0)$ and note that $\Phi_{\varepsilon} \leq(2 \pi \varepsilon)^{-1 / 2}$. This, in conjunction with the dominated convergence theorem and the fundamental theorem of calculus easily yields (2.2) and (2.3). Statement (2.4) follows easily from the approximate identity property of the $\Phi_{\varepsilon}$ on $C_{b}(\mathbb{R})$.

It is worthwhile to note that $\partial G \underline{\mu} / \partial x$ need not exist in general, but does if $\mu$ has a bounded density function [8, p. 303]. By (2.3) then, $G \underline{\mu}$ is $C^{1}$ if $\mu$ has a continuous density function. 
If $g$ is any function on $\mathbb{R} \times \mathbb{R}_{+}$we define $H^{*}(g)$, the reduite of $g$, by $H^{*}(g)=\inf \{f: f \geq g$ and $f$ is superparabolic $\}$. Let

$$
H(x, t)=\liminf _{(y, s) \rightarrow(x, t)} H^{*}(y, s)
$$

be the lower regularization of $H^{*}$. We are interested in the choice $g=G \mu-G \underline{\nu}$, which is a continuous function and is bounded on the set $\{(x, t): t<\bar{T}\}$ for any $T>0$.

Lemma 2.2. Let $g$ be a continuous function having at least one superparabolic majorant and $H=H(g)$ the lower regularization of its reduite. Then $H \geq g$. Moreover,

$$
H \text { is continuous and superparabolic on } \mathbb{R} \times(0, \infty) \text {, }
$$

and

$$
D \equiv\{H>g\} \text { is open }
$$

Proof. Since $g$ has a superparabolic majorant $H^{*}$ is not identically infinite, so $H$ is superparabolic [8, 1.XVII.13, p. 314]. We have $H \geq g$ since $H^{*} \geq g$ and $g$ is continuous. Since $H$ is superparabolic it is lower semicontinuous. Since $H-g$ is also lower semicontinuous we have (2.6).

Let $\sigma$ be the Riesz measure of $H$. Suppose $\sigma(D)>0$. Then by writing $D=\bigcup_{q \in \mathbb{Q}}\{H>q>g\}$ it is easy to see that there is a nonempty rectangle $R=[a, b] \times\left[t_{0}, t_{1}\right) \subseteq D$ and a constant $c$ so that $\sigma\left((a, b) \times\left(t_{0}, t_{1}\right)\right)>0$ and $H>c>g$ on $R$. Define a function $\tau_{R} H$ to agree with $H$ off $R$ and be given on $R$ by the solution of the heat equation on $R$ with the values of $H$ prescribed on the lower boundary of $R$, i.e., that part of $\partial R$ which is included in $R$. Then by [8, pp. 280-281] the function $\tau_{R} H$ is superparabolic and $\tau_{R} H \leq H$. By the maximum principle [8, p. 279] we have $\tau_{R} H \geq c>g$ on $R$, hence $\tau_{R} H \geq g$ everywhere. But $\tau_{R} H\left(x, t_{1}\right)<H\left(x, t_{1}\right), a<x<b$, since $\sigma(R)>0$ implies that $H$ is not parabolic on $R$. (See statement (b) on p. 280 of [8].) This contradicts the minimality of $H^{*}$. Thus $\sigma(D)=0$ and (2.7) is proved.

Finally, $H$ is continuous, and therefore (2.5) holds, by the Evans-Vasilesco Theorem, since $H=g$ (continuous) on $D^{c} \supset$ support $(\sigma)$. (See pp. 64-65 of [8]. The proof given there carries over with some minor changes to the parabolic context.)

From now on we will denote by $H$ the lower regularization of the reduite of $g=G \underline{\mu}-G \underline{\nu}$. Since $G \underline{\mu}$ is a superparabolic majorant of $g$, it follows from lemma 2.2 that $H$ satisfies (2.5)-(2.7). It will eventually be shown, under the additional hypotheses of Theorem 1.1, that $D$ has the form $D=$ $\left\{(x, t):-s_{-}(t)<x<s_{+}(t), t>0\right\}$, where $s_{ \pm}$are as in Theorem 1.1. 
To motivate the introduction of $H$, we remark that under suitable smoothness and other assumptions on a function $g$, the function $F=H(g)$ satisfies

$$
\begin{gathered}
\frac{1}{2} \partial^{2} F / \partial x^{2}=\partial F / \partial t \quad \text { on } D=\{F>g\}, \\
F=g \text { on } \partial D,
\end{gathered}
$$

and

$$
\partial F / \partial x=\partial g / \partial x \text { on } \partial D \text {. }
$$

This is another kind of free boundary problem, with the extra boundary condition (2.10) playing the role of condition (1.4). In fact, such free boundary problems have been widely studied and arise, for example, in optimal stopping and stochastic control theory (see $\S 5$ for further discussion.) There is a formal transformation due to Schatz [22] which converts a solution of $(2.8)-(2.10)$ into a solution of a corresponding Stefan problem. Study of this transformation suggests that the function $u$ of Theorem 1.1 should be given by

$$
u=\partial U / \partial t \text { on } D \text { and } u=0 \text { elsewhere, }
$$

where $U=H-g$. It will be proved below that $u$ has the required properties, but first we need more information on the structure of $D$.

Theorem 2.3. There exists a nonnegative upper semicontinuous function $\varphi$ having no strict local minima such that

$$
D=\{(x, t): t>\varphi(x)\},
$$

and

$$
x \in \operatorname{support}(\mu) \Rightarrow \text { there exist } x_{n} \rightarrow x \text { with } \varphi\left(x_{n}\right)>0 .
$$

Moreover, if we express support $(\mu)^{c}=\bigcup_{j=1}^{\infty} I_{j}$ as a disjoint union of maximal open intervals $I_{j}$, then

$$
\nu\left(I_{j}\right)>0 \Rightarrow \varphi \equiv 0 \text { on } I_{j} \text {, while } \nu\left(I_{j}\right)=0 \Rightarrow \varphi>0 \text { on } I_{j} .
$$

Proof. We begin by proving that

$$
U(x, t)=H(x, t)-g(x, t) \text { is nondecreasing in } t \text { for each } x .
$$

Since $D=\{(x, t): U(x, t)>0\}$, we would then deduce that $D$ has the form (2.12) for some upper semicontinuous function $\varphi$.

Now the semigroup property of $\Phi_{\varepsilon}(x)=G(0, \varepsilon ; x, 0)$ implies that $g=$ $G \underline{\mu}-G \underline{\nu}$ satisfies the identity

$$
g(x, t+s)=g(x, t)+\Phi_{t} * g(x, s), \quad t, s>0,
$$

where the convolution is taken only on the spatial variable. Let $H_{s}(x, t)=$ $H(x, t+s)$, a superparabolic function. Since $\Phi_{t} * g(x, s)$ is a parabolic function of $(x, t)$ we see that $H_{s}(x, t)-\Phi_{t} * g(x, s)$ is also superparabolic. By $(2.16)$ we 
have $H_{s}(x, t)-\Phi_{t} * g(x, s) \geq g(x, t)$ and, therefore, $H_{s}(x, t)-\Phi_{t} * g(x, s) \geq$ $H(x, t)$. Thus for $s>0$

$$
\frac{H(x, t+s)-H(x, t)}{s} \geq \frac{1}{s} \Phi_{t} * g(x, s) .
$$

Since $\Phi_{t}(\cdot) \in C_{b}(\mathbb{R})$ it follows from (2.4) and the mean value theorem that

$$
\lim _{s \rightarrow 0} \frac{1}{s} \Phi_{t} * g(x, s)=\Phi_{t} *(\mu-\nu)(x)=\frac{\partial g}{\partial t}(x, t)
$$

If $(x, t)$ belongs to $D$ (where $H$ is differentiable) we conclude from (2.17) and $(2.18)$ that $\partial U(x, t) / \partial t \geq 0$. Since $U$ is continuous and vanishes off $D$, we may conclude that $(2.15)$ holds.

We now turn to the proof of (2.13). Suppose this were false. Then we could find $a<b<x<c<d$ such that $\mu((b, c))>0, \nu([a, d])=0$ (see (2.1)) and $H$ is parabolic in $R=(a, d) \times(0, \infty)$. Since $H$ clearly satisfies $-G \underline{\nu} \leq H \leq G \underline{\mu}$ we have

$$
|H(x, t)| \leq(\pi / 2)^{-1 / 2}(\|\mu\|+1) t^{1 / 2} .
$$

Since both $H$ and $G \underline{\nu}$ are parabolic in $R$ and may be represented there in terms of their boundary values, it is easy to see that there are constants $A$ and $B$ depending only on $\|\mu\|$ and $\delta=(d-c) \wedge(b-a)$ such that

$$
|H(x, t)| \leq A e^{-B / t}, \quad 0<t \leq 1, b \leq x \leq c,
$$

and

$$
G \underline{\nu}(x, t) \leq A e^{-B / t}, \quad 0<t \leq 1, b \leq x \leq c .
$$

Now for $0<t \leq 1$ and $y \in(b, c)$ we also have

$$
\begin{aligned}
\int_{b}^{c} \Phi_{t}(y-\eta) d \eta & \geq \int_{0}^{(c-b) / 2} \Phi_{t}(\xi) d \xi \\
& \geq \frac{1}{2}-(2 \pi)^{-1 / 2} \int_{(c-b) / 2}^{\infty} e^{-\xi^{2} / 2} d \xi \\
& \geq \varepsilon=\varepsilon(c-b)>0 .
\end{aligned}
$$

It follows that we have

$$
\int_{b}^{c} G \underline{\mu}(\eta, t) d \eta \geq \mu((b, c)) \varepsilon t, \quad 0<t \leq 1 .
$$

But (2.20)-(2.22) are clearly incompatible as $t \rightarrow 0$ with $H \geq g$, and this contradiction completes the proof of (2.13).

To prove (2.14), first let $I=I_{j}$ be such that $\nu(I)>0$. Let $T>0$ and $R=I \times[0, T)$. Then $g$ is bounded, continuous and subparabolic on $R$. Let $\psi$ be the solution of the heat equation in $R$ with boundary values given by the restriction of $g$ to the lower boundary of $R$. Then for any $x \in I$ we have $H(x, T) \geq \psi(x, T)>g(x, T)$, the strict inequality holding since $g$ is not 
parabolic in $R$. Since $T$ was arbitrary we have $I \times(0, \infty) \subseteq D$, i.e., $\varphi \equiv 0$ on $I$.

Now suppose $\nu(I)=0$. We shall suppose that $I$ is a finite interval, say $I=(a, b)$, the case of a half-line being handled by a similar argument. Since $I$ is maximal we have $\{a, b\} \subseteq \operatorname{support}(\mu)$. Suppose $c<d$ satisfy $\varphi(c) \wedge \varphi(d)=$ $\delta>0$ and $\nu([c, d])=0$. Let $R=[c, d] \times[0, \delta)$. Then $U=H-g$ is nonnegative subparabolic on $R$ and vanishes on the lower boundary of $R$. It follows that $U$ vanishes identically on $R$, hence $\varphi(x) \geq \delta$ for $x \in I$. By (2.1) and (2.13) the interval $(a, b)$ may be covered by a union of such $(c, d)$.

There remains only to show that $\varphi$ can have no strict local minima. Suppose $x$ were a strict local minimum of $\varphi$. Then $x \notin \operatorname{support}(\nu)$ by $(2.1)$ and (2.14). Accordingly, we can find $a<x<b$ with $\varphi(x)<\varphi(a) \wedge \varphi(b)$ and $\nu([a, b])=$ 0 . Then $U$ is nonnegative subparabolic on $\{(y, t): a<y<b, \varphi(y)<t \leq$ $\varphi(a) \wedge \varphi(b)\}$ and vanishes on the lower boundary of this set. Thus $U$ vanishes identically there, contradicting $\varphi(x)<\varphi(a) \wedge \varphi(b)$. This completes the proof of Theorem 2.3.

Remark 2.4. Suppose $\nu$ has compact support as it does under the full hypotheses of Theorem 1.1. Then an argument similar to the one given at the end of the proof of Theorem 2.3 shows that if $\operatorname{support}(\nu) \subseteq(a, b)$ then $\varphi$ is monotone nondecreasing on $(b, \infty)$ and nonincreasing on $(-\infty, a)$. For example, if $c \geq b$, one may rule out the possibility that $\varphi$ is monotone nonincreasing on $[c, \infty)$ with $\lim _{x \rightarrow \infty} \varphi(x)<\varphi(c)$ by the same subparabolic argument applied to the domain $\{(x, t): c<x<\infty, \varphi(x)<t \leq \varphi(c)\}$. Thus, since $\varphi$ has no strict local minima, it follows that $\varphi$ must be monotone nondecreasing on $[b, \infty)$.

Let $u$ be defined as in (2.11) above and put

$$
u^{*}(x, t)=\int G_{D}(x, t ; \eta, 0) \nu(d \eta) .
$$

Note that $u^{*}$ is parabolic in $D$ and vanishes continuously at $\partial_{+} D=$ $\partial D \cap\{(x, t): t>0\}$. This follows since each point $(\xi, s) \in \partial_{+} D$ is regular for $D^{c}$. Indeed, the line segment $\{\xi\} \times[0, s]$ is contained in $D^{c}$ and $(\xi, s)$ is regular for this line segment. have

Extending $u^{*}$ to all of $\mathbb{R} \times(0, \infty)$ by defining it to be 0 on $D^{c}$, we then

$$
u^{*} \text { is continuous on } \mathbb{R} \times(0, \infty)
$$

We also have

$$
u^{*}(x, t) \rightarrow \nu \quad \text { in } C_{b}^{*}(\mathbb{R}) \text { as } t \rightarrow 0 .
$$

This follows from the easily proved fact that $G_{D}(x, t ; \eta, 0)-\Phi_{t}(x-\eta) \rightarrow 0$ as $t \rightarrow 0$ uniformly for $\eta$ belonging to support $(\nu),($ See $(2.1)$ and (2.14)), and the fact that $\Phi_{t} * \nu(x) \rightarrow \nu$ in $C_{b}^{*}(\mathbb{R})$ as $t \rightarrow 0$.

For the remainder of this section we assume that $\mu$ and $\nu$ satisfy all of the hypotheses of Theorem 1.1 and that (2.1) continues to hold. 
Theorem 2.5. We have $u=u^{*}$.

We prove Theorem 2.5 below after we show how to complete the proof of Theorem 1.1 under condition (2.1).

Let $\left(-c_{-}, c_{+}\right)$be the largest open interval containing 0 such that

$$
\mu\left(\left(-c_{-}, c_{+}\right)\right)=0 .
$$

Then we have $b_{ \pm} \leq c_{ \pm}$and by Remark $2.4 \varphi$ is positive and nondecreasing on $\left(c_{+}, \infty\right)$. Let $s_{+}$be the left-continuous inverse of $\varphi$ on $\left(c_{+}, \infty\right)$, i.e., $s_{+}(t)=\inf \left\{x \geq c_{+}: \varphi(x) \geq t\right\}$, with the usual convention that $\inf (\varnothing)=\infty$. Similarly, define $s_{-}$by $-s_{-}(t)=\sup \left\{x \leq-c_{-}: \varphi(x) \geq t\right\}$. Then $s_{ \pm}$are extended real-valued, left-continuous, and $s_{ \pm}\left(0_{+}\right)=c_{ \pm} \geq b_{ \pm}$. We also have $(x, t) \in D \Leftrightarrow t>\varphi(x) \Leftrightarrow x \in\left(-s_{-}(t), s_{+}(t)\right), t>0$. The values $s_{ \pm}(0)$ may be chosen arbitrarily.

Assuming the result of Theorem 2.5, we must only prove that the integral equations (1.7) hold in order to complete the proof of Theorem 1.1.

Lemma 2.6. $H$ is the heat potential of the measure $\underline{\mu}_{D} c$, i.e., $H(x, t)=$ $G\left(\underline{\mu}{ }_{D} c\right)(x, t)$.

Proof. Since $H$ satisfies $0 \leq H \leq\|\mu\| t^{1 / 2}$ it follows that 0 is the greatest parabolic minorant of $H$. Since $H$ is parabolic in $D$ and superparabolic, the Riesz decomposition implies that $H=G \sigma$, where $\sigma$ is a positive measure on $\mathbb{R} \times \mathbb{R}_{+}$satisfying $\sigma(D)=0$. We claim further that

$$
\sigma(\partial D)=0 \text {. }
$$

To see this, let $\mathscr{V}$ be any open subset of $\mathbb{R} \times \mathbb{R}_{+}$such that $\underline{\nu}(\mathscr{V})=0$. It then suffices to show $\sigma(\partial D \cap \mathscr{V})=0$, by (2.14). Now $U=H-G \underline{\mu}+G \underline{\nu}$ is subparabolic on $\mathscr{V}$ since $\underline{\nu}(\mathscr{V})=0$, and $U=0$ on $D^{c} \cap \mathscr{V}$. Therefore the restriction of the measure $L(U)$ to $\mathscr{V}$ is a positive measure, and hence, $0 \leq\left.\sigma\right|_{\mathscr{V}} \leq\left.\underline{\mu}\right|_{\mathscr{V}}$. But (1.5) implies that $\underline{\mu}(\partial D)=0$, hence (2.25) follows.

Since $H=g$ on $D^{c}$, it is easy to show that the restriction of $\sigma$ to the exterior of $D$ (i.e., the complement of the closure of $D$ ) agrees with $L(g)=-\mu$ there. Combining this with $\sigma(D)=0$ and (2.25) we conclude that $\sigma=\underline{\mu} 1_{D} c$, and the proof of Lemma 2.6 is complete.

We shall also need the following facts:

$$
\int_{0}^{T} u(x, t) d t=U(x, T)
$$

and

$$
\int_{0}^{T} \int_{\mathbb{R}}(|x|+1) u(x, t) d x d t<\infty, \quad 0<T<\infty .
$$

The first follows from the definition $(2.11),(2.23)$ and the bound $0 \leq u(x, t) \leq$ $(2 \pi t)^{-1 / 2}$, a consequence of Theorem 2.5. The second follows from Theorem 2.5 and Fubini's theorem. 
Next, we prove

$$
\int_{-s_{-}(t)}^{s_{+}(t)} u(x, t) d x=1-\int_{-s_{-}(t)}^{s_{+}(t)} \mu(d x), \quad 0<t<\infty .
$$

By (2.26) and Fubini's theorem, for $T>0$,

$$
\int_{0}^{T} \int_{-s_{-}(t)}^{s_{+}(t)} u(x, t) d t=\int_{\mathbb{R}} \int_{\varphi(x)}^{T} u(x, t) d t d x=\int_{\mathbb{R}}(H-g)(x, T) d x
$$

But, by Lemma 2.6, $(H-g)(x, t)=G \underline{\nu}(x, t)-G\left(\underline{\mu} 1_{D}\right)(x, t)$, hence

$$
\begin{aligned}
\int_{\mathbb{R}}(H-g)(x, T) d x & =\int_{\mathbb{R}} G \underline{\nu}(x, T) d x-\int_{\mathbb{R}} G\left(\underline{\mu} 1_{D}\right)(x, T) d x \\
& =T-\int_{0}^{T} \int_{-s_{-}(t)}^{s_{+}(t)} \mu(d \xi) .
\end{aligned}
$$

Thus,

$$
\int_{0}^{T} \int_{-s_{-}(t)}^{s_{+}(t)} u(x, t) d x d t=T-\int_{0}^{T} \int_{-s_{-}(t)}^{s_{+}(t)} \mu(d x) .
$$

Differentiating both sides of this equation with respect to $T$ we find that $(2.28)$ holds for almost every $t$. But the left-hand side of $(2.28)$ is a continuous function of $t$ (since $u$ vanishes on $\partial_{+} D$ ) and the right-hand side is left continuous. It follows that $(2.28)$ holds for all $0<t<\infty$.

We now prove

$$
\int_{-s_{-}(t)}^{s_{+}(t)} x u(x, t) d x=\int x \nu(d x)-\int_{-_{-}(t)}^{s_{+}(t)} x \mu(d x), \quad 0<t<\infty .
$$

Combining (2.28) and (2.29) yields (1.7).

Let $T_{*}=\inf \left\{t: s_{+}(t) \wedge s_{-}(t)=\infty\right\}$. If $T_{*}<\infty$ and $s_{+}\left(T_{*}\right) \wedge s_{-}\left(T_{*}\right)<\infty$ then (2.29) holding for $t>T_{*}$ follows from (2.29) for $t=T_{*}$, since (2.28) implies $\mu\left(\left(s_{+}\left(T_{*}\right), \infty\right)\right)=\mu\left(\left(-\infty,-s_{-}\left(T_{*}\right)\right)=0\right.$. In all other cases, the validity of (2.29) for $t \geq T_{*}$ follows from its validity for $t<T_{*}$, together with either the monotone convergence theorem or the interpretation of $\int x \mu(d x)$ in the improper sense. Because of these observations, we may suppose that one or both of the endpoints of integration in (2.29) is finite, and, therefore, the integrals there are well defined.

By (2.26), (2.27) and Fubini's theorem

$$
\begin{aligned}
\int_{0}^{T} & \int_{-s_{-}(t)}^{s_{+}(t)} x u(x, t) d x d t=\int_{\mathbb{R}} x \int_{\varphi(x)}^{T} u(x, t) d t d x \\
& =\int_{\mathbb{R}} x(H-g)(x, T) d x \\
& =\int_{\mathbb{R}} x G \underline{\nu}(x, T) d x-\int_{\mathbb{R}} x G\left(\underline{\mu} 1_{D}\right)(x, T) d x \\
& =T \int_{\mathbb{R}} \xi \nu(d \xi)-\int_{0}^{T} \int_{-s_{-}(t)}^{s_{+}(t)} \xi \mu(d \xi),
\end{aligned}
$$


the last equality following from $\int x \Phi_{T}(x-\xi) d x=\xi$. Then (2.29) follows by differentiation as before.

We now turn to the proof of Theorem 2.5, and begin by establishing the $a$ priori bound

$$
0 \leq u(x, t) \leq G \nu(x, t)=\Phi_{t} * \nu(x), \quad(x, t) \in D .
$$

Let $D_{s}=\{x:(x, s) \in D\}$. Then by Lemma 2.6 (whose proof did not assume the result of Theorem 2.5)

$$
\begin{aligned}
H(x, t+s) & =\int_{0}^{t} \int_{D_{u}^{c}} \Phi_{u}(x-y) \mu(d y) d u+\int_{t}^{t+s} \int_{D_{u}^{c}} \Phi_{u}(x-y) \mu(d y) d u \\
& =H(x, t)+\int_{0}^{s} \int_{D_{v+t}^{c}} \Phi_{v+t}(x-y) \mu(d y) d v \\
& \leq H(x, t)+\int_{0}^{s} \int_{D_{v}^{c}} \Phi_{v+t}(x-y) \mu(d y) d v,
\end{aligned}
$$

since for $t>0$ we have $D_{v+t}^{c} \subseteq D_{v}^{c}$. Thus $H(x, t+s) \leq H(x, t)+\Phi_{t} *$ $H(x, s) \leq H(x, t)+\Phi_{t} * G \underline{\mu}(x, s)$. Subtracting identity (2.16) from both sides of this inequality and using (2.15) we conclude that $0 \leq U(x, t+s)-U(x, t) \leq$ $\Phi_{t} * G \underline{\nu}(x, s)$. By the same argument that yielded $(2.18)$ we have that on $D$ (where $U$ is differentiable with respect to $t$ ) $0 \leq \frac{\partial U}{\partial t}(x, t) \leq G \nu(x, t)$, which proves $(2.30)$.

\section{Lemma 2.7. We have}

$$
G \underline{\mu}(x, t)=o\left(t^{1 / 2}\right) \text { as } t \rightarrow 0 \text {, uniformly in } x .
$$

Proof. We may assume $\mu \neq 0$. Let $\varepsilon>0$ be given and choose $N$ so large that

$$
e^{-N^{2} / 2} \leq \frac{\sqrt{\pi}\|\mu\|^{-1} \varepsilon}{2 \sqrt{2}}
$$

and then $\Delta>0$ so small that

$$
\mu([x-N \sqrt{\Delta}, x+N \sqrt{\Delta}])<\frac{\sqrt{\pi} \varepsilon}{2 \sqrt{2}}, \quad x \in \mathbb{R} .
$$

The latter is possible by (1.5). Then for $0<t \leq \Delta$ we have

$$
\begin{aligned}
\int_{0}^{t} \int_{\mathbb{R}} \Phi_{s}(x-y) \mu(d y) d s \\
=\int_{0}^{t} \int_{|x-y| \leq N \sqrt{t}} \Phi_{s}(x-y) \mu(d y) d s \\
\quad+\int_{0}^{t} \int_{|x-y|>N \sqrt{t}} \Phi_{s}(x-y) \mu(d y) d s \\
\equiv \mathrm{I}+\mathrm{II} .
\end{aligned}
$$


By (2.33),

$$
\begin{aligned}
I & \leq \int_{0}^{t} \int_{|x-y| \leq N \sqrt{t}}(2 \pi s)^{-1 / 2} \mu(d y) d s \\
& =\int_{0}^{t}(2 \pi s)^{-1 / 2} \mu([x-N \sqrt{t}, x+N \sqrt{t}]) d s \\
& <\frac{\varepsilon}{2} \sqrt{t} .
\end{aligned}
$$

By (2.32),

$$
\begin{aligned}
\mathrm{II} & \leq \int_{0}^{t} \int_{\mathbb{R}}(2 \pi s)^{-1 / 2} e^{-N^{2} t / 2 s} \mu(d y) d s \\
& \leq e^{-N^{2} / 2}\|\mu\| \int_{0}^{t}(2 \pi s)^{-1 / 2} d s \\
& \leq \frac{\varepsilon}{2} \sqrt{t} .
\end{aligned}
$$

Combining the two estimates completes the proof of Lemma 2.7.

Lemma 2.8. Let $(x, t) \in \partial_{+} D$. Then

$$
U(x, s+t)-U(x, t)(=U(x, s+t))=o(s) \text { as } s \rightarrow 0 .
$$

Moreover, for fixed $\delta>0$, this estimate is uniform in $(x, t) \in \partial_{+} D$ satisfying $t \geq \delta$.

Proof. Let $\tau_{s}=\inf \{u>0:(x-B(u), t+s-u) \notin D\}$. (We suppress the dependence of $\tau_{s}$ on $(x, t)$.) Note that $\tau_{s} \leq t+s$, a.s.

We claim that

$$
E\left[g\left(x-B\left(\tau_{s}\right), t+s-\tau_{s}\right) ; \tau_{s}>t\right]=o(s),
$$

uniformly in $(x, t)$. Since $\tau_{s}<t+s$ implies $x-B\left(\tau_{s}\right) \notin \operatorname{support}(\nu),(2.21)$ and Lemma 2.7 imply $\mid g\left(\left(x-B\left(\tau_{s}\right), t+s-\tau_{s}\right) \mid=o\left(s^{1 / 2}\right)\right.$ uniformly a.s. on $\left\{\tau_{s}>t\right\}$. Thus, to show (2.34), it is enough to show that $P_{(x, t)}\left(\tau_{s}>t\right)=O\left(s^{1 / 2}\right)$ uniformly in $x$ and $t \geq \delta$ for each $\delta>0$. But since the segment $\{x\} \times[0, t]$ is contained in $D^{c}$, such an estimate would follow from the Brownian probability estimate

$$
P_{0}(B(u) \neq 0, s \leq u \leq t) \leq C(t) s^{1 / 2},
$$

with $C(t)$ a constant depending only on $t$.

To prove (2.35), first note that for $z \neq 0$ and $s \leq t / 2$,

$$
\begin{gathered}
P_{z}(B(u) \neq 0,0 \leq u \leq t-s)=P_{0}\left(\sup _{0 \leq u \leq t-s} B(u) \leq z\right) \\
=\int_{-|z|}^{|z|} \Phi_{t-s}(\xi) d \xi \leq 2|| \Phi_{t-s} \|_{\infty}|z|=C_{1}(t)|z|,
\end{gathered}
$$


where $C_{1}(t)$ depends only on $t$, by symmetry of Brownian motion and the reflection principle. (See e.g., [13, p. 26].) By the Markov property

$$
\begin{aligned}
P_{0}(B(u) \neq 0, s \leq u \leq t) & =\int_{\mathbb{R}} \Phi_{s}(z) P_{z}(B(u) \neq 0,0 \leq u \leq t-s) \\
& \leq C_{1}(t) \int_{\mathbb{R}} \Phi_{s}(z)|z| d z=C_{2}(t) s^{1 / 2}
\end{aligned}
$$

where $C_{2}(t)$ depends only on $t$. Thus (2.35) holds for $s \leq t / 2$. Then we may choose $C(t) \geq C_{2}(t)$ sufficiently large so that $(2.35)$ holds for all $s$.

Now $H\left(\left(x-B\left(\tau_{s} \wedge u\right), t+s-\tau_{s} \wedge u\right)\right.$ is a uniformly integrable martingale indexed by $u$. Thus, letting $u \rightarrow \infty$ we have

$$
\begin{aligned}
H(x, t+s) & =E_{0} H\left(x-B\left(\tau_{s}\right), t+s-\tau_{s}\right) \\
& =E_{0} g\left(x-B\left(\tau_{s} \wedge t\right), t+s-\tau_{s} \wedge t\right)+o(s),
\end{aligned}
$$

where we used (2.34) and its proof in the last step.

Now, since $H$ is superparabolic, and supermartingale expectations decrease,

$$
\begin{aligned}
g(x, t) & =H(x, t) \geq E H\left(x-B\left(\tau_{s} \wedge t\right), t-\tau_{s} \wedge t\right) \\
& \geq E g\left(x-B\left(\tau_{s} \wedge t\right), t-\tau_{s} \wedge t\right) .
\end{aligned}
$$

Combining this with the identity (2.16),

$$
\begin{aligned}
& H(x, t+s)-H(x, t) \\
& \quad \leq E\left\{g\left(x-B\left(\tau_{s} \wedge t\right), t+s-\tau_{s} \wedge t\right)-g\left(x-B\left(\tau_{s} \wedge t\right), t-\tau_{s} \wedge t\right)\right\}+o(s) \\
& \quad=E \Phi_{t-\tau_{s} \wedge t} * g\left(x-B\left(\tau_{s} \wedge t\right), s\right)+o(s)=\Phi_{t} * g(x, s)+o(s),
\end{aligned}
$$

where the last line follows from the strong Markov property. Since $U=H-g$ the desired result now follows from (2.16).

To continue the proof of Theorem 2.5, fix $(x, t) \in D$ and choose $0<\eta<t$. Since $G \nu$ is bounded on $\{(y, t): t \geq \eta\}$, by (2.30) there is a constant $A=A(\eta)$ such that

$$
0 \leq u(y, s) \leq A, \quad s \geq \eta
$$

If $(y, s) \in \partial_{+} D$ then (2.36) and the definition of $u$ imply that $U(y, s+\delta)=$ $\int_{0}^{\delta} u(y, s+r) d r$. Combining this with Lemma 2.8 and Chebyshev's inequality we can find $\delta>0$ (depending on $\eta$ and $\varepsilon$ ) so that

$$
|\{v \in(0, \delta): u(y, s+v)>\varepsilon\}|<\varepsilon \delta, \quad(y, s) \in \partial D, \quad s>\eta,
$$

and so that $\delta<(t-\eta) \wedge \operatorname{dist}((x, t), \partial D)$.

Then with $\tau=\inf \{v:(x+B(v), t-v) \notin D\} \wedge(t-\eta)$,

$$
\begin{aligned}
\int_{0}^{\delta} u(x, t+r) d r= & \int_{0}^{\delta} E_{0} u(x+B(\tau), t+r-\tau) d r \\
= & \int_{\mathbb{R}} \int_{0}^{\delta} u(y, \varphi(y)+r) d r P(x+B(\tau) \in d y, \tau<t-\eta) \\
& +\int_{D_{\eta}} \int_{0}^{\delta} u(z, \eta+r) P(x+B(\tau) \in d z, \tau=t-\eta) .
\end{aligned}
$$


(Note that $\tau$ does not depend on $r$.) By (2.36) and (2.37), $\varphi(y)>\eta$ implies $\int_{0}^{\delta} u(y, \varphi(y)+r) d r \leq \varepsilon \delta A+\varepsilon \delta$. It follows that

$$
\begin{aligned}
u(x, t) & =\lim _{\delta \rightarrow 0}(1 / \delta) \int_{0}^{\delta} u(x, t+r) d r \\
& =\int_{D_{\eta}} u(y, \eta) P(x+B(\tau) \in d y, \tau=t-\eta) \\
& =\int_{D_{\eta}} u(y, \eta) G_{D}(x, t ; y, \eta) d y .
\end{aligned}
$$

Now let $K=\operatorname{support}(\nu)$. Let $\mathscr{O}_{1}$ and $\mathscr{O}_{2}$ be open subsets of $\mathbb{R}$ such that $K \subseteq \mathscr{O}_{1} \subset \overline{\mathscr{O}}_{1} \subset\left(-b_{-}, b_{+}\right), \mathscr{O}_{2} \cap K=\varnothing$, and $\mathscr{O}_{1} \cup \mathscr{O}_{2}=\mathbb{R}$. It follows from (2.30) that

$$
\int_{\mathscr{O}_{2}} u(y, \eta) d y \rightarrow 0 \text { as } \eta \rightarrow 0
$$

and

$$
\int_{\mathbb{R}} u(y, \eta) d y \leq\|\nu\|
$$

Also

$$
\begin{aligned}
& \int_{\mathscr{O}_{1}} G \mu(y, \eta) d y \rightarrow 0 \quad \text { as } \eta \rightarrow 0, \\
& \int_{\mathscr{O}_{1}}\left|H_{t}(y, \eta)\right| d y \rightarrow 0 \quad \text { as } \eta \rightarrow 0 .
\end{aligned}
$$

(The latter follows easily from an examination of the Poisson representation of $H$ on the slab $\left(-b_{-}, b_{+}\right) \times(0, \infty)$ where it is parabolic.) It is easy to show that

(2.42) $\quad G_{D}(x, t ; y, \eta) \rightarrow G_{D}(x, t ; y, 0)$ as $\eta \rightarrow 0$, uniformly in $y \in \mathscr{O}_{1}$.

We will now show that

$$
\int_{D_{\eta}} u(y, \eta) G_{D}(x, t ; y, \eta) d y \rightarrow \int G_{D}(x, t ; y, 0) \nu(d y) .
$$

To see this, it suffices, in view of (2.38), (2.39), (2.42) and a partition of unity argument, to show that

$$
\int u(y, \eta) G_{D}(x, t ; y, 0) \psi(y) d y \rightarrow \int G_{D}(x, t ; y, 0) \psi(y) \nu(d y)
$$

for any bounded continuous function supported in $\mathscr{O}_{1}$. Since $u=H_{t}-G \mu+G \nu$ on $D$, this follows from $(2.40),(2.41)$, and

$$
\int G \nu(y, \eta) G_{D}(x, t ; y, 0) \psi(y) d y \rightarrow \int G_{D}(x, t ; y, 0) \psi(y) \nu(d y),
$$

as $\eta \rightarrow 0$. 
The desired result, $u(x, t)=u^{*}(x, t)$ follows, since the right-hand side of (2.43) is equal to $u^{*}(x, t)$.

\section{UNIQUENESS}

The purpose of this section is to prove Theorem 1.2. Throughout the section we shall suppose that $\nu$ is a probability measure, as a convenient normalization. Suppose $\mu$ and $\nu$ satisfy the hypotheses of Theorem 1.2 and $\left(u, s_{-}, s_{+}\right)$is a triple satisfying the various conditions listed there. We wish to show that $u, s_{-}$, and $s_{+}$are uniquely determined by $\mu$ and $\nu$. This will be done by completing the following outline.

Step 1. Let $D=\left\{(x, t):-s_{-}(t)<x<s_{+}(t), 0<t<\infty\right\}$. The set $D$ is open since $s_{ \pm}$are left-continuous. Let $u^{*}(x, t)=\int G_{D}(x, t ; \eta, 0) \nu(d \eta)$ where $G_{D}$ is the parabolic Green function (or killed Brownian transition function) of $D$. We will show that the functions $u$ and $u^{*}$ are identical, and hence $u$ is uniquely determined, provided $s_{ \pm}$are uniquely determined.

Step 2. Here, and throughout the rest of this section $\tau$ denotes the exit time of co-space-time Brownian motion from $D$, i.e.,

$$
\tau=\inf \left\{t>0: B(0)+B(t) \notin\left(-s_{-}(t), s_{+}(t)\right)\right\} .
$$

(We assume the motion starts from time 0 here.) Let $\sigma(d x)=P_{\nu}(B(\tau) \in d x)$ be the exit-position distribution. We will prove that $\sigma=\left.\mu\right|_{(A, B)}$, where $A=$ $-s_{-}(\infty)$, and $B=s_{+}(\infty)$.

Step 3. We will prove that $(A, B)$ is uniquely determined by the conditions of the theorem. Thus, by Step 2, $\sigma$ is determined. (We will in fact prove slightly less in the case $\|\mu\|=\|\nu\|$.)

Step 4. We will show that for each given exit-distribution $\sigma$, there is at most one pair of functions $s_{ \pm}$, satisfying the conditions of Theorem 1.2, which give rise to that $\sigma$.

The results of Steps 1-3, taken together, show that the conditions of the Theorem uniquely determine the exit distribution from $D$ of co-space-time Brownian motion started at time 0 with spatial distribution $\nu$. Hence, in light of the result of Step 4, the boundaries $s_{ \pm}$are uniquely determined.

We now proceed to fill in the details of each step.

Step 1 (details). Because $u$ is continuous on $\mathbb{R} \times(0, \infty)$, parabolic on $D$, and (1.11) holds, a Phragmén-Lindelöf type extension of the maximum principle (see, e.g., [28, Theorem 6.1]) shows that we have

$$
u(x, t)=\int G_{D}(x, t ; \eta, \delta) u(\eta, \delta) d \eta
$$

for $0<\delta \leq t$ and each $\delta>0$. (Condition (1.11) is needed in order to handle the possibility that $D \cap\{(y, s): \delta<s<t\}$ is unbounded.) Choose nonnegative 
continuous functions $\psi$ and $\chi$ such that $\psi+\chi \equiv 1, \psi$ is compactly supported in $\left(-s_{-}(0), s_{+}(0)\right)$, and $\psi=1$ (hence $\left.\chi=0\right)$ on $\left(-s_{-}(0)+\varepsilon, s_{+}(0)-\right.$ $\varepsilon$ ) for some $\varepsilon>0$. It is easy to show that for fixed $(x, t)$, the function $G_{D}(x, t ; \eta, \delta)$ converges to $G_{D}(x, t ; \eta, 0)$ as $\delta \rightarrow 0$, uniformly for $\eta$ belonging to compact subsets of $\left(-s_{-}(0), s_{+}(0)\right)$. Therefore $\psi(\eta) G_{D}(x, t ; \eta, \delta) \rightarrow$ $\psi(\eta) G_{D}(x, t ; \eta, 0)$ uniformly in $\eta$ as $\delta \rightarrow 0$. By (1.6),

$$
\begin{aligned}
\lim _{\delta \rightarrow 0} \int & u(\eta, \delta) G_{D}(x, t ; \eta, \delta) \psi(\eta) d \eta \\
= & \lim _{\delta \rightarrow 0} \int u(\eta, \delta) G_{D}(x, t ; \eta, 0) \psi(\eta) d \eta \\
= & \int G_{D}(x, t ; \eta, 0) \psi(\eta) \nu(d \eta) .
\end{aligned}
$$

On the other hand, $\chi(\eta) G_{D}(x, t ; \eta, \delta) \leq[2 \pi(t-\delta)]^{-1 / 2} \chi(\eta)$, so that

$$
\begin{aligned}
\lim _{\delta \rightarrow 0} & \sup \int u(\eta, \delta) G_{D}(x, t ; \eta, \delta) \chi(\eta) d \eta \\
& \leq(2 \pi t)^{-1 / 2} \lim _{\delta \rightarrow 0} \int \chi(\eta) u(\eta, \delta) d \eta \\
& =(2 \pi t)^{-1 / 2} \int \chi(\eta) \nu(d \eta) .
\end{aligned}
$$

This latter integral may be made arbitrarily small by taking $\varepsilon$ small. Since $\varepsilon$ was arbitrary, we have that

$$
\begin{aligned}
\lim _{\delta \rightarrow 0} \int & u(\eta, \delta) G_{D}(x, t ; \eta, \delta) d \eta \\
& =\int G_{D}(x, t ; \eta, 0) \nu(d \eta) \\
& =u^{*}(x, t) .
\end{aligned}
$$

Thus, by (3.1), we conclude that $u=u^{*}$, and Step 1 is complete.

Step 2 (details). Let $F_{\mu}$ denote the distribution function of $\mu$, i.e., $F_{\mu}(x)=$ $\mu([0, x))$ for $x>0$ and $F_{\mu}(x)=-\mu([x, 0))$ for $x<0$. Similarly, define $F_{\sigma}(x)$ in terms of $\sigma$. We begin by showing that the nondecreasing functions $s_{ \pm}$of Theorem 1.2 are actually strictly increasing on the sets where they are finite. The assumption that $F_{\mu}$ be continuous is crucial in this result.

Lemma 3.1. Let $T_{+}=\sup \left\{t: s_{+}(t)<\infty\right\}$. Then $s_{+}$is strictly increasing on $\left(0, T_{+}\right)$(A similar result holds for $\left.s_{-}.\right)$

Proof. Suppose there were a nonempty interval $\left(t_{1}, t_{2}\right)$ on which $s_{+}(t)=\alpha<$ $\infty$. We shall assume that $s_{-}$is finite on $\left(t_{1}, t_{2}\right)$. (The case in which $s_{-}=\infty$ on a nonempty subinterval of $\left(t_{1}, t_{2}\right)$ is easier to handle.) Fix a point $t \in\left(t_{1}, t_{2}\right)$ at which $s_{-}$is differentiable. (Almost every point in $\left(t_{1}, t_{2}\right)$ has this property.) 
For any $\delta<t_{2}-t$ we have by (1.7)

$$
\begin{aligned}
\int_{I(t+\delta)}(x+\beta) u(x, t+\delta) d x-\int_{I(t)}(x+\beta) u(x, t) d x \\
=-\int_{-s_{-}(t+\delta)}^{-s_{-}(t)}(x+\beta) \mu(d x),
\end{aligned}
$$

where $I(t)=\left[-s_{-}(t), s_{+}(t)\right]$.

By [5, Theorem 14.4.1] the function $u_{x}$ has a continuous extension to the line-segment $L=\{\alpha\} \times\left(t_{1}, t_{2}\right)$. Therefore, we may apply Green's formula on the rectangle $\left[-s_{-}(t)+\delta, \alpha\right] \times[t, t+\delta]$. (We assume from now on that $\delta$ satisfies $0<\delta<\left(\alpha+s_{-}(t)\right) \wedge\left(t_{2}-t\right)$.) Applying Green's formula with $v=x+\beta$ and using (3.2) we derive

$$
\begin{aligned}
\int_{t+\delta}^{t}(\alpha+\beta) u_{x}(\alpha, \tau) d \tau+2 \int_{-s_{-}(t)}^{s_{-}(t)+\delta}(x+\beta) u(x, t) d x \\
\quad-2 \int_{-s_{-}(t+\delta)}^{-s_{-}(t)+\delta}(x+\beta) u(x, t+\delta) d x \\
\quad-2 \int_{-s_{-}(t+\delta)}^{-s_{-}(t)}(x+\beta) \mu(d x)+\int_{t}^{t+\delta}\left(-s_{-}(t)+\delta+\beta\right) u_{x}\left(-s_{-}(t)+\delta, \tau\right) d \tau \\
\quad-\int_{t}^{t+\delta} u\left(-s_{-}(t)+\delta, \tau\right) d \tau=0 .
\end{aligned}
$$

We now choose $\beta=s_{-}(t)-\delta$, so that the fifth integral above vanishes. By the mean value theorem the first integral becomes $-(\beta+\alpha) \delta u_{x}\left(\alpha, \tau_{*}\right)$, for some $\tau_{*} \in(t, t+\delta)$. Each of the remaining integrals is easily seen to be $o(\delta)$ as $\delta \rightarrow 0$, by using the facts that $u$ vanishes on $\partial D, s_{-}$is differentiable at $t$, and $\mu$ has a continuous distribution function. It follows that $u_{x}(\alpha, t)=0$. Since this reasoning applies for almost every $t \in\left(t_{1}, t_{2}\right)$, and $u_{x}$ is continuous on $L$, we conclude that $u_{x}$ vanishes identically on $L$. But this is impossible, since $u$ does not vanish identically. (For example, reflection across $L$ would give a parabolic function having an interior minimum.) This completes the proof of Lemma 3.1 .

Lemma 3.1 has two important consequences: $F_{\sigma}$ is continuous, and the triple $\left(u, s_{-}, s_{+}\right)$satisfies $(1.7)$ with $\mu$ replaced by $\sigma$. The continuity of $F_{\sigma}$ follows easily from the continuity of the distribution function of $\tau$ and from the strict increase of $s_{ \pm}$. To see the former, note that $P_{\nu}(\tau \in[t, t+\varepsilon)) \leq$ $P_{\nu}\left(B(\tau) \notin\left(-s_{-}(\tau), s_{+}(\tau)\right)\right.$ for some $\tau \in[t, t+\varepsilon)$, and $\left.B(t) \in\left(-s_{-}(t), s_{+}(t)\right)\right)$. The latter probability tends to 0 as $\varepsilon$ tends to 0 by path continuity of Brownian motion. 
To see that (1.7) holds with $\sigma$ in place of $\mu$, fix $t>0$ and note that $M(h)=B(t \wedge h)+\beta$ is a uniformly integrable martingale. Therefore

$$
\begin{aligned}
\int(x+\beta) \nu(d x) & =E_{\nu} M(0)=E_{\nu} M(\tau)=E_{\nu}(M(t) ; \tau \geq t)+E_{\nu}(M(\tau) ; \tau<t) \\
& =\int_{-s_{-}(t)}^{s_{+}(t)}(x+\beta) u(x, t) d x+\int_{-s_{-}(t)}^{s_{+}(t)}(x+\beta) \sigma(d x) .
\end{aligned}
$$

(Note that we have used the equality $\{\tau<t\}=\left\{B(\tau) \in\left(-s_{-}(t), s_{+}(t)\right)\right\}$, a consequence of the strict increase of $s_{ \pm}$.)

It follows easily from (1.7) and the fact that $u$ vanishes on $\partial D$ that

$$
\mu\left(\left(s_{+}(t), s_{+}(t+)\right)\right)=\mu\left(\left(-s_{-}(t+),-_{-}(t)\right)\right)=0 \text { for every } t .
$$

The same clearly holds for $\sigma$ since Brownian motion cannot leave $D$ via the jumps in $s_{ \pm}$. Thus we may conclude that the functions $F_{\mu}\left(s_{+}(t)\right)-F_{\sigma}\left(s_{+}(t)\right)$ and $F_{\mu}\left(-s_{-}(t)\right)-F_{\sigma}\left(-s_{-}(t)\right)$ are continuous and vanish at $t=0$.

We are now ready to complete Step 2 by showing that $\left.\mu\right|_{(A, B)}=\sigma$. The argument is similar to the proof of Gronwall's Lemma. First, since both $\mu$ and $\sigma$ satisfy (1.7) we have

$\int_{-_{-}(t)}^{s_{+}(t)}(\alpha x+\beta) \mu(d x)=\int_{-s_{-}(t)}^{s_{+}(t)}(\alpha x+\beta) \sigma(d x), \quad$ for all $\alpha, \beta \in \mathbb{R}, 0<t<\infty$.

Integration by parts yields

$$
\begin{aligned}
& \left(\alpha s_{+}(t)+\beta\right) F_{\mu}\left(s_{+}(t)\right)+\left(\alpha s_{-}(t)-\beta\right) F_{\mu}\left(-s_{-}(t)\right)-\alpha \int_{-s_{-}(t)}^{s_{+}(t)} F_{\mu}(x) d x \\
& =\left(\alpha s_{+}(t)+\beta\right) F_{\sigma}\left(s_{+}(t)\right)+\left(\alpha s_{-}(t)-\beta\right) F_{\sigma}\left(-s_{-}(t)\right)-\alpha \int_{-s_{-}(t)}^{s_{+}(t)} F_{\sigma}(x) d x .
\end{aligned}
$$

Taking $\alpha=0$ and $\beta=1$ gives

$$
F_{\mu}\left(s_{+}(t)\right)-F_{\mu}\left(-s_{-}(t)\right)=F_{\sigma}\left(s_{+}(t)\right)-F_{\sigma}\left(-s_{-}(t)\right),
$$

and taking $\alpha=1$ and $\beta=0$ gives

$$
\begin{aligned}
& s_{+}(t) F_{\mu}\left(s_{+}(t)\right)+s_{-}(t) F_{\mu}\left(-s_{-}(t)\right)-\int_{-s_{-}(t)}^{s_{+}(t)} F_{\mu}(x) d x \\
& =s_{+}(t) F_{\sigma}\left(s_{+}(t)\right)+s_{-}(t) F_{\sigma}\left(-s_{-}(t)\right)-\int_{-s_{-}(t)}^{s_{+}(t)} F_{\sigma}(x) d x .
\end{aligned}
$$

Multiplying both sides of (3.3) by $s_{+}(t)$ and subtracting from (3.4), and then multiplying (3.3) by $s_{-}(t)$ and adding to (3.4) yields

$$
\begin{aligned}
F_{\mu}\left(s_{+}(t)\right) & -F_{\sigma}\left(s_{+}(t)\right)=F_{\mu}\left(-s_{-}(t)\right)-F_{\sigma}\left(-s_{-}(t)\right) \\
& =\left[1 /\left(s_{+}(t)+s_{-}(t)\right)\right] \int_{-s_{-}(t)}^{s_{+}(t)}\left(F_{\mu}(x)-F_{\sigma}(x)\right) d x .
\end{aligned}
$$

Recall that $F_{\mu}\left(s_{+}(t)\right)-F_{\sigma}\left(s_{+}(t)\right)$ is a continuous function of $t$ vanishing at 0 . If this function were not identically zero then we could find $T>0$ such that 
$\left|F_{\mu}\left(s_{+}(t)\right)-F_{\sigma}\left(s_{+}(t)\right)\right|$ would be strictly maximized on $[0, T]$ at $t=T$. Since $\mu$ and $\sigma$ do not charge the jumps of $s_{+}$we would also have $\left|F_{\mu}(x)-F_{\sigma}(x)\right| \leq$ $\left|F_{\mu}\left(s_{+}(T)\right)-F_{\sigma}\left(s_{+}(T)\right)\right|$ for $0 \leq x<s_{+}(T)$. But then substituting $t=T$ in (3.5) we obtain a contradiction.

We conclude that $F_{\mu}\left(s_{ \pm}(t)\right)=F_{\sigma}\left(s_{ \pm}(t)\right)$ for all $t>0$, and hence $F_{\mu}(x)=$ $F_{\sigma}(x)$ for all $x \in(A, B)$. Of course, this equality of distribution functions implies the desired result: $\sigma=\left.\mu\right|_{(A, B)}$.

Step 3 (details). First we show that if $\mu(\mathbb{R})<\nu(\mathbb{R})$ then $A=-\infty$ and $B=\infty$. If either one of $A$ or $B$, say $A$, were finite, then by the maximum principle, $u(x, t) \leq \int\left\{\Phi_{t}(x-y)-\Phi_{t}(2 A-x-y)\right\} \nu(d y)$ for $A<x$, and it is easy to conclude that $\int_{A}^{B} u(x, t) d x \rightarrow 0$ as $t \rightarrow \infty$. Therefore, by (1.7),

$$
\int_{A}^{B} \mu(d x)=\int_{A}^{B} \nu(d x)=\nu(\mathbb{R}) .
$$

But this is impossible, since $\mu((A, B)) \leq \mu(\mathbb{R})<\nu(\mathbb{R})$. (The quantity $\lim _{t \rightarrow \infty} \int u(x, t) d x$ makes up the "deficit" $\nu(\mathbb{R})-\mu(\mathbb{R})$.)

Now suppose $\mu(\mathbb{R})=\nu(\mathbb{R})$. Then we will prove that $(A, B)$ contains $(\alpha, \beta)$, where $\alpha=\inf \operatorname{support}(\mu)$ and $\beta=\sup \operatorname{support}(\mu)$. If $(\alpha, \beta)=$ $(-\infty, \infty)$, then we necessarily have $(A, B)=(-\infty, \infty)$, by (3.6) and the argument which lead to (3.6). On the other hand, if $\beta$ is finite and $B \leq \beta$ then again (3.6) holds and forces the inequality $B \geq \beta$. Therefore, we must have $B \geq \beta$ in any case. (It is easy to show, based upon the result of Step 2, that $B$ can take only the values $\beta$ or $+\infty$ and that $A$ can take only the values $\alpha$ or $-\infty$. It is possible to show that if $\mu$ and $\nu$ have the same mean then necessarily $(A, B)=(\alpha, \beta)$, but the other possibilities can occur if the means differ. See Theorem 6.3 below.)

For the remainder of our discussion of Step 3 we shall suppose $\nu(\mathbb{R})<\mu(\mathbb{R})$ and prove that the pair $(A, B)$ is uniquely determined. First note that at least one of $A$ or $B$ must be finite. This is so because (1.7) implies the inequality $\mu(A, B) \leq \nu(\mathbb{R})$. Therefore, we have (3.6) holding.

Lemma 3.2. Suppose $\mu$ is a positive measure with a continuous distribution function and we have

$$
\int_{A_{1}}^{B_{1}}(\alpha x+\beta) \mu(d x)=\int_{A_{2}}^{B_{2}}(\alpha x+\beta) \mu(d x), \quad \alpha, \beta \in \mathbb{R},
$$

where $-\infty \leq A_{i}<B_{i} \leq \infty$ for $i=1,2$. (It is assumed that the integrals exist and that $\mu\left(A_{i}, B_{i}\right)$ is finite.) Suppose also that

$$
\mu\left(\left(B_{i}-\varepsilon, B_{i}\right)\right)>0 \text { and } \mu\left(\left(A_{i}, A_{i}+\varepsilon\right)\right)>0 \text { for each } \varepsilon>0 .
$$

(If $B_{i}=+\infty$ this is interpreted to mean that $\mu((N, \infty))>0$ for every $N$, with an analogous interpretation if $A_{i}=-\infty$.) Then $A_{1}=A_{2}$ and $B_{1}=B_{2}$.

Proof. Suppose $\left(A_{1}, B_{1}\right) \neq\left(A_{2}, B_{2}\right)$. Taking $\alpha=0$ in (3.7) we conclude that $\mu\left(A_{1}, B_{1}\right)=\mu\left(A_{2}, B_{2}\right)$ and hence neither of the intervals $\left[A_{i}, B_{i}\right]$ can contain 
the other. Taking $\beta=0$ in (3.7) shows that the intervals cannot be disjoint. Therefore we may suppose that $A_{1}<A_{2}<B_{1}<B_{2}$. But clearly

$$
1 / \mu\left(\left(A_{1}, B_{1}\right)\right) \int_{A_{1}}^{B_{1}} x \mu(d x)<1 / \mu\left(\left(A_{2}, B_{1}\right)\right) \int_{A_{2}}^{B_{1}} x \mu(d x)
$$

and

$$
1 / \mu\left(\left(A_{2}, B_{1}\right)\right) \int_{A_{2}}^{B_{1}} x \mu(d x)<1 / \mu\left(\left(A_{2}, B_{2}\right)\right) \int_{A_{2}}^{B_{2}} x \mu(d x) .
$$

Since $\mu\left(\left(A_{1}, B_{1}\right)\right)=\mu\left(\left(A_{2}, B_{2}\right)\right)$ we conclude that $\int_{A_{1}}^{B_{1}} x \mu(d x)<\int_{A_{2}}^{B_{2}} x \mu(d x)$, contradicting (3.7). Therefore $\left(A_{1}, B_{1}\right)=\left(A_{2}, B_{2}\right)$, and the proof is complete.

It follows easily from Lemma 3.2 that there is a unique pair $\left(A_{1}, B_{1}\right)$ such that conditions (3.8) hold, and also

$$
\mu\left(\left(A_{1}, B_{1}\right)\right)=\nu(\mathbb{R}),
$$

and

$$
\left|\int_{A_{1}}^{B_{1}} x \mu(d x)-\int x \nu(d x)\right| \text { is as small as possible. }
$$

We will prove that $\left(A_{1}, B_{1}\right)=(A, B)$. First, suppose that $A>A_{1}$. Then $A$ is finite, so by (1.7) we have $\int_{A}^{B}(x-A) \mu(d x) \leq \int(x-A) \nu(d x)$. Thus $\int_{A}^{B} x \mu(d x) \leq \int x \nu(d x)$ by (3.6), and so, combining (3.10), (3.9) and (3.6) we have

$$
\int_{A}^{B} x \mu(d x) \leq \int_{A_{1}}^{B_{1}} x \mu(d x) .
$$

If equality holds in (3.11) then we are done by Lemma 3.2, so we may suppose that strict inequality holds there. It follows that $B=\infty$ since otherwise (1.7) and the dominated convergence theorem would imply that equality holds in (3.11). But $A$ satisfies (3.8) by the result of Step 2, hence

$$
\int_{A}^{B} x \mu(d x)=\int_{A}^{\infty} x \mu(d x)>\int_{A_{1}}^{\infty} x \mu(d x) \geq \int_{A_{1}}^{B_{1}} x \mu(d x),
$$

contradicting (3.11). We conclude that $A \leq A_{1}$.

Similarly, one may prove that $B \geq B_{1}$. Combining this with (3.6), (3.9), and (3.8) shows that $(A, B)=\left(A_{1}, B_{1}\right)$.

Step 4 (details). Suppose $s_{ \pm}$and $\sigma_{ \pm}$are two pairs of functions satisfying the hypotheses of Theorem 1.2 with the same $\mu$ and $\nu$. Let $D$ and $\Delta$ be the domains in space-time defined by $s_{ \pm}$and $\sigma_{ \pm}$respectively. Suppose $D \neq \Delta$. Then we will show that the exit distributions of co-space-time Brownian motion from $D$ and $\Delta$ differ. More precisely, if $\tau$ and $\tau^{*}$ denote the respective exit times, then we shall construct a set $E$ such that $P_{\nu}(B(\tau) \in E) \neq P_{\nu}\left(B\left(\tau^{*}\right) \in E\right)$. We distinguish two cases. 
Case 1. Either $D$ contains $\Delta$ or $\Delta$ contains $D$.

For definiteness, let us suppose $\Delta$ strictly contained in $D$, i.e., we have $s_{ \pm}(t) \geq$ $\sigma_{ \pm}(t)$ for all $t$ and $s_{+}\left(t_{0}\right)>\sigma_{+}\left(t_{0}\right)$ for some $t_{0}$. Take $E=\left[-\sigma_{-}\left(t_{0}\right), \sigma_{+}\left(t_{0}\right)\right]^{c}$. Then it is easy to show that

$$
\left\{B\left(\tau^{*}\right) \in E\right\} \subseteq\{B(\tau) \in E\} .
$$

By left-continuity of $s_{+}$and the fact that $\sigma_{+}$is nondecreasing, there exists $\delta>0$ such that $\sigma_{+}(t)<s_{+}(t), t_{0}-\delta \leq t \leq t_{0}$. Then we have $P_{\nu}(B(\tau) \in E$, $\left.B\left(\tau^{*}\right) \notin E\right) \geq P_{\nu}\left(B(\tau) \in E, t_{0}-\delta \leq \tau^{*} \leq t_{0}, B\left(\tau^{*}\right)=\sigma_{+}\left(\tau^{*}\right)\right)$, and the latter probability is easily seen to be strictly positive. Combining this observation with (3.12), we have $P_{\nu}(B(\tau) \in E)>P_{\nu}\left(B\left(\tau^{*}\right) \in E\right)$. This completes the proof in Case 1.

Case 2. The remaining case.

For definiteness, we shall suppose that there are $t_{0}$ and $t_{1}$ with $s_{+}\left(t_{0}\right)>\sigma_{+}\left(t_{0}\right)$ and $s_{+}\left(t_{1}\right)<\sigma_{+}\left(t_{1}\right)$. (There are actually 8 possibilities obtained by independently choosing + or - and interchanging $s$ and $\sigma$ in each of the two inequalities. Each of them may be handled by an argument similar to the one we give.) Let $E=\left\{x\right.$ : for some $t, s_{+}(t+) \geq x \geq \sigma_{+}(t)$ and $s_{+}(t)>$ $\left.\sigma_{+}(t)\right\} \cup\left\{x\right.$ : for some $t,-s_{-}(t+) \leq x \leq-\sigma_{-}(t)$ and $\left.-s_{-}(t)<-\sigma_{-}(t)\right\}$. Then $E$ is not empty since $s_{+}\left(t_{0}\right)$ belongs to $E$. We shall show that

$$
\{B(\tau) \in E\} \subseteq\left\{B\left(\tau^{*}\right) \in E\right\} .
$$

The idea is that in order for the Brownian path to exit $D$ at a point of $E$ it must have already exited $\Delta$. Moreover the first such exit from $\Delta$ must have occurred at a point of $E$, since, at that time the path had not yet exited from $D$. Proceeding more formally, first note that $B(\tau) \in E$ implies $\tau>\tau^{*}$. Indeed, suppose also that $B(\tau)=s_{+}(\tau)$. Then there exists a $t$ such that $\sigma_{+}(t) \leq B(\tau) \leq$ $s_{+}(t+)$ and $\sigma_{+}(t)<s_{+}(t)$. Clearly, $\tau \leq t$. If $\tau=t$ then the assertion follows since $B(\tau)=s_{+}(\tau)>\sigma_{+}(\tau)$. If $\tau<t$ then $\sigma_{+}(\tau)<\sigma_{+}(t)$ (by Lemma 3.1) $\leq$ $B(\tau)$ and the assertion again follows. The argument in the case $B(\tau)=-s_{-}(\tau)$ is similar. Now let $G=\left\{x\right.$ : for some $\left.t, \sigma_{+}(t)=x \geq s_{+}(t)\right\} \cup\{x$ : for some $t$, $\left.-\sigma_{-}(t)=x \leq-s_{-}(t)\right\}$. Then $B\left(\tau^{*}\right) \in G$ implies $\tau^{*} \geq \tau$. Therefore, supposing $B(\tau) \in E$, we cannot have $B\left(\tau^{*}\right) \in G$. Thus, either $B\left(\tau^{*}\right)=\sigma_{+}\left(\tau^{*}\right)<s_{+}\left(\tau^{*}\right)$ or $B\left(\tau^{*}\right)=-\sigma_{-}\left(\tau^{*}\right)>-s_{-}\left(\tau^{*}\right)$. In either case we have $B\left(\tau^{*}\right) \in E$ and so (3.13) holds.

Now by left continuity of the $s_{ \pm}$and $\sigma_{ \pm}$there is $\delta>0$ such that $\sigma_{+}(t)<$ $s_{+}(t), t_{0}-\delta \leq t \leq t_{0}$, and $s_{+}(t)<\sigma_{+}(t), t_{1}-\delta \leq t \leq t_{1}$. Now $P_{\nu}(B(\tau) \notin E$, $\left.B\left(\tau^{*}\right) \in E\right) \geq P_{\nu}\left(t_{1}-\delta \leq \tau \leq t_{1}, B(\tau)>0, t_{0}-\delta \leq \tau^{*} \leq t_{0}, B\left(\tau^{*}\right)>0\right)$, and the latter probability is easily seen to be strictly positive. Together with (3.13) this shows that $P_{\nu}\left(B\left(\tau^{*}\right) \in E\right)>P_{\nu}(B(\tau) \in E)$, and the proof is complete.

\section{Existence (GENERAL CASE)}

In this section we complete the proof of Theorem 1.1 by removing assumption (2.1). We need the following monotonicity result. 
Lemma 4.1. Suppose $\left(\nu_{i}, \mu_{i}\right), i=1,2$, are two pairs of measures satisfying the hypotheses of Theorem 1.1 and having disjoint supports. Suppose $\mu_{1} \leq \mu_{2}$ and $\nu_{1} \geq \nu_{2}$. Let $\left(u_{i}, s_{-}^{(i)}, s_{+}^{(i)}\right)$ be the unique solutions of the Stefan problem with data $\left(\nu_{i}, \mu_{i}\right)$ whose existence has been proved in $\S 2$. Then $s_{ \pm}^{(2)} \leq s_{ \pm}^{(1)}$ and $u_{2} \leq u_{1}$.

Proof. We need only prove the former inequality since the latter follows readily with the aid of the maximum principle. The proof is very similar to Step 4 in the proof of Theorem 1.2. Define $D_{i}$ by $D_{i}=\left\{(x, t):-s_{-}^{(i)}(t)<x<s_{+}^{(i)}(t), 0<\right.$ $t<\infty\}$ for $i=1,2$. Suppose $D_{2}$ is not contained in $D_{1}$. Then we consider two cases.

Case 1. $D_{1}$ is properly contained in $D_{2}$.

Choose $t$ such that $\left(-s_{-}^{(1)}(t), s_{+}^{(1)}(t)\right) \equiv E$ is properly contained in $\left(-s_{-}^{(2)}(t)\right.$, $\left.s_{+}^{(2)}(t)\right)$. Let $\tau_{i}$ be the exit times of co-space-time Brownian motion from $D_{i}$. Then $\left\{B\left(\tau_{2}\right) \in E\right\} \subseteq\left\{B\left(\tau_{1}\right) \in E\right\}$. For any $x$ such that $P_{x}\left(B\left(\tau_{1}\right) \in E\right)>0$ it is easy to show that $P_{x}\left(B\left(\tau_{1}\right) \in E, B\left(\tau_{2}\right) \notin E\right)>0$. Therefore,

$$
\begin{aligned}
\int P_{x}\left(B\left(\tau_{2}\right) \in E\right) \nu_{2}(d x) & \leq \int P_{x}\left(B\left(\tau_{2}\right) \in E\right) \nu_{1}(d x) \\
& <\int P_{x}\left(B\left(\tau_{1}\right) \in E\right) \nu_{1}(d x) .
\end{aligned}
$$

By the result of Step 2 in the proof of Theorem 1.2 we have

$$
\mu_{2}(E)=P_{\nu_{2}}\left(B\left(\tau_{2}\right) \in E\right)<P_{\nu_{1}}\left(B\left(\tau_{1}\right) \in E\right)=\mu_{1}(E),
$$

contradicting the assumption that $\mu_{1} \leq \mu_{2}$.

Case 2. The remaining case.

As in Case 2 of Step 4 in the proof of Theorem 1.2 we suppose for definiteness that there are $t_{0}$ and $t_{1}$ with $s_{+}^{(2)}\left(t_{0}\right)>s_{+}^{(1)}\left(t_{0}\right)$ and $s_{+}^{(2)}\left(t_{1}\right)<s_{+}^{(1)}\left(t_{1}\right)$. Let $E=$ $\left\{x\right.$ : for some $t, s_{+}^{(2)}(t+) \geq x \geq s_{+}^{(1)}(t)$, and $\left.s_{+}^{(2)}(t)>s_{+}^{(1)}(t)\right\} \cup\{x$ : for some $t$, $-s_{-}^{(2)}\left(t^{+}\right) \leq x \leq-s_{-}^{(1)}(t)$, and $\left.-s_{-}^{(2)}(t)<s_{-}^{(1)}(t)\right\}$. Then exactly as in $\S 3$ we derive $P_{x}\left(B\left(\tau_{1}\right) \in E\right)>P_{x}\left(B\left(\tau_{2}\right) \in E\right)$ for any $x$ in support $\left(\nu_{1}\right)$. Then, as in Case 1, $\mu_{2}(E)=\int P_{x}\left(B\left(\tau_{2}\right) \in E\right) \nu_{2}(d x) \leq \int P_{x}\left(B\left(\tau_{2}\right) \in E\right) \nu_{1}(d x)<P_{\nu_{1}}\left(B\left(\tau_{1}\right) \in E\right)=$ $\mu_{1}\left(E \cap\left(-s_{-}^{(1)}(\infty), s_{+}^{(1)}(\infty)\right)\right) \leq \mu_{1}(E)$, a contradiction which completes the proof of Lemma 4.1.

To finish the proof of Theorem 1.1, let $\delta>0$ and $\left(v, \sigma_{-}, \sigma_{+}\right)$be the solution of the Stefan problem with data $\left(\nu, \mu 1_{\left(-b_{-}-\delta, b_{+}+\delta\right)}\right)$, and let $\left(u_{\varepsilon}, s_{-}^{(\varepsilon)}, s_{+}^{(\varepsilon)}\right)$ be the solutions with data $\left(\nu 1_{\left(-b_{-}+\varepsilon, b_{+}-\varepsilon\right)}, \mu\right)$. Then by Lemma 4.1 , the $s_{ \pm}^{(\varepsilon)}$ increase as $\varepsilon \downarrow 0$ to left-continuous nondecreasing functions $s_{ \pm}$satisfying $s_{ \pm} \leq \sigma_{ \pm}$. Let $\tau$ denote the exit time of co-space-time Brownian motion from the space-time region $D$ bounded by $-s_{-}$and $s_{+}$and $\tau^{\varepsilon}$ the exit times from the regions bounded by $-s_{-}^{(\varepsilon)}$ and $s_{+}^{(\varepsilon)}$. If $\tau(\omega)<\infty$ then compactness 
of the co-space-time Brownian path on $[0, \tau(\omega)]$ easily implies that we have $\tau^{\varepsilon}(\omega) \rightarrow \tau(\omega)$ as $\varepsilon \rightarrow 0$. If $\tau(\omega)=\infty$ the same conclusion follows from the compactness of the path on $[0, N]$ for any $N$. It follows that the measures $\left.P \nu\right|_{\left(-b_{-}+\varepsilon, b_{+}-\varepsilon\right)}\left(B\left(\tau^{\varepsilon}\right) \in d x\right)$ converge in $C_{b}^{*}(\mathbb{R})$ to $P_{\nu}(B(\tau) \in d x)$. But the former measures equal $\left.\mu\right|_{\left(-s_{-}^{(\varepsilon)}(\infty), s_{+}^{(\varepsilon)}(\infty)\right)}$ by the results of $\S 3$, and it follows that $P_{\nu}(B(\tau) \in d x)=\left.\mu\right|_{\left(-s_{-}(\infty), s_{+}(\infty)\right)}$.

Let $D=\left\{(x, t): t>0\right.$ and $\left.-s_{-}(t)<x<s_{+}(t)\right\}$ and

$$
u(x, t)=\int G_{D}(x, t ; y, 0) \nu(d y) .
$$

To show that $\left(u, s_{+}, s_{-}\right)$is the desired solution of the Stefan problem it suffices to show that the integral equations are satisfied. For this, it follows that

$$
\begin{aligned}
\int(x+\beta) \nu(d x) & =E_{\nu} M(0)=E_{\nu} M(t) \\
& =E_{\nu}(M(\tau) ; \tau \leq t)+E_{\nu}(M(t), t<\tau) \\
& =\int_{-s_{-}(t)}^{s_{+}(t)}(x+\beta) \mu(d x)+\int_{-s_{-}(t)}^{s_{+}(t)}(x+\beta) u(x, t) d x, \\
& \text { where } M(t)=B(t \wedge \tau)+\beta,
\end{aligned}
$$

which is the desired result.

\section{Applications}

As mentioned in the introduction, our approach to the Stefan problem has been motivated by the work of Rost and Meyer on the filling scheme, and by the recent application of their ideas to the Skorohod embedding problem by P. Chacon. Recall that the object of the Skorohod embedding problem is to find a stoping time $\tau$ of Brownian motion for which the equality of measures $P_{\nu}(B(\tau) \in d x)=\mu(d x)$ holds, where $\mu$ and $\nu$ are given probability measures. This result, under a variety of more or less restrictive hypotheses, has been obtained by numerous authors. The following partial list shows the wide variety of methods used: [1-4, 6, 7, 9, 11, 12, 14-20, and 25].

On the other hand, our results yield an independent proof of P. Chacon's result for Brownian motion in nearly its full generality (Chacon's hypotheses on $\mu$ and $\nu$ are slightly less restrictive, and he considers a class of Markov processes which includes Brownian motion among others).

Theorem 5.1 (P. Chacon [6]). Let $\mu$ and $\nu$ be probability measures which satisfy the hypotheses of Theorem 1.1. Let $\left(u, s_{-}, s_{+}\right)$be the solution to the Stefan problem of Theorem 1.1. Then the stopping time

$$
\tau=\inf \left\{t: B(t) \notin\left(-s_{-}(t), s_{+}(t)\right)\right\}
$$

solves the Skorohod embedding problem with initial distribution $\nu$ and target measure $\mu$, i.e., $P_{\nu}(B(\tau) \in d x)=\mu(d x)$. 
Theorem 5.1 follows immediately from Theorem 1.1 and Step 2 in the proof of Theorem 1.2.

Our results also have applications to optimal stopping problems for Brownian motion. Let $T>0$ and $\gamma(x, t)$ be a bounded measurable function on $\mathbb{R} \times$ $[0, T]$. Define

$$
\hat{\gamma}(x, T)=\sup E_{x} \gamma(B(\tau), T-\tau),
$$

where the supremum is taken over Brownian stopping times $\tau$ which satisfy $\tau \leq T$, a.s. The optimal stopping problem with payoff function $\gamma$ and time horizon $T$ has two parts:

(I) Compute $\hat{\gamma}$ in terms of $\gamma$, and,

(II) given $x$, find, if possible, a stopping time $\tau \leq T$ such that $\hat{\gamma}(x, T)=$ $E_{x} \gamma(B(\tau), T-\tau)$.

For example, in some simplistic models of stock market trading $\gamma(B(s), T-s)$ may represent the value at time $s$ of a portfolio held by an investor who has agreed to sell his portfolio on or before some future date $T$. The time $\tau$ would then be the time the investor should sell so as to maximize his expected income.

Let us assume for simplicity that $\gamma$ is continuous. Towards the solution of (I) we then have the following well-known characterization of $\hat{\gamma}$ :

Proposition 5.1. The function $\hat{\gamma}$ is the least superparabolic majorant of $\gamma$ on $\mathbb{R} \times[0, T]$. Moreover, $\hat{\gamma}$ is continuous, and parabolic on the set $C=\{\hat{\gamma}>\gamma\}$.

Given the set $C$ one may then solve problem (II) by taking $\tau \wedge T$ as the optimal stopping time, where $\tau$ denotes the exit time from $C$ of space-time Brownian motion. For this reason the set $C$ is usually called the continuation region. The function $\hat{\gamma}$ is termed the reward function.

We may view the function $H$ of $\S 2$ as the reward function for an optimal stopping problem involving space-time Brownian motion.

Theorem 5.2. Let $\mu$ and $\nu$ be as in Theorem 1.1. Set $\gamma(x, T)=G \mu(x, T)-$ $G \underline{\nu}(x, T)$. Then $\hat{\gamma}(x, T)=H(x, T)$ is the reward function corresponding to the payoff function $\gamma$. The continuation region is the set $D$ of $\S 2$.

Van Moerbeke has shown that in certain cases $\hat{\gamma}$ may be obtained as the solution of a free boundary problem for the heat equation with prescribed flux.

Theorem 5.3 [26, §2.8]. Suppose $\gamma$ is $C^{2}$ with bounded second derivatives on $\mathbb{R} \times[0, T]$. Suppose also that the continuation region is bounded by one or more continuous curves which are piecewise $C^{1}$. Then $\hat{\gamma}$ satisfies

$$
\partial \hat{\gamma} / \partial x=\partial \gamma / \partial x \text { at regular points of } \partial C \text {. }
$$

(For simplicity we state this result with stronger boundedness assumptions than are needed in [26].)

Van Moerbeke goes on to point out that if $C$ is bounded by two piecewise $C^{1}$ curves $-s_{-}$and $s_{+}$, then the triple $\left(u, s_{-}, s_{+}\right)$satisfies the classical Stefan 
problem (1.1)-(1.4) on $[0, T]$ with $h$ there replaced by the function of two variables $h(x, t)=L(\gamma)(x, t), \varphi(x)=h(x, 0)$, and $u$ is obtained by the "Schatz transformation" [22] $u(x, t)=\partial(\hat{\gamma}-\gamma) / \partial t$.

The ad hoc smoothness hypothesis on $\partial C$ somewhat limits the usefulness of Theorem 5.3, although it may be verified in a number of interesting cases [26, especially, $\S \S 3.2$ and $4 ; 27]$. In the situation of Theorem 5.2, Theorem 6.1 below implies that $s_{ \pm}$are $C^{1}$ in case $\mu$ has a continuous density function which is strictly positive on $\left(-b_{-}, b_{+}\right)^{c}$, and by $[8$, p. 303] the function $\gamma$ has the required smoothness provided also that $\mu$ and $\nu$ have Lipschitz continuous density functions. Thus, under these conditions, the result of Theorem 5.2 follows from that of Theorem 5.3.

Payoff functions of the form $G \underline{\mu}-G \underline{\nu}$ may seem rather special, but they do correspond to a very natural class of optimal stopping problems. For example, suppose $\mu$ and $\nu$ have density functions $h$ and $\rho$, and consider the problem of maximizing the expression

$$
E_{x} \int_{0}^{\tau}\{\rho(B(s))-h(B(s))\} d s
$$

over stopping times $\tau \leq T$. The identities

$$
\begin{gathered}
E_{x}(G \underline{\mu}-G \underline{\nu})(B(\tau), T-\tau)=E_{x} \int_{0}^{T-\tau} E_{B(\tau)}[h(B(s))-\rho(B(s))] d s \\
=G_{\underline{\mu}}(x, T)-G_{\underline{\nu}}(x, T)+E_{x} \int_{0}^{\tau}[\rho(B(s))-h(B(s))] d s,
\end{gathered}
$$

which follow from the strong Markov property, show that the set $D$ is also the continuation region for this problem. For more general measures $\mu$ and $\nu$ the same is true provided we replace (5.2) by $E_{x} \int_{\mathbb{R}} \Omega(\tau, y)[\nu(d y)-\mu(d y)]$, where $\Omega(t, x)$ is the jointly continuous Brownian local time. (See [13, Chapter 2] for properties of local time.)

In summary, we have the following result.

Theorem 5.4. Let $\mu$ and $\nu$ satisfy the hypotheses of Theorem 1.1 and let $T>0$ be given. Then for each $x$ the stopping time $\tau$ which maximizes $E_{x} \int_{\mathbb{R}} \Omega(\tau, y)[\nu(d y)-\mu(d x)]$ and satisfies $\tau \leq T$, a.s., is given by $\tau=$ $\inf \left\{t: x+B(t) \notin\left(-s_{-}(T-t), s_{+}(T-t)\right)\right\}$, where $s_{ \pm}$are the free boundaries of Theorem 1.1 .

\section{Properties of THe FRee boundaries}

If $\mu$ has a density function $h$, the smoothness properties of $h$ should be reflected in smoothness properties of the free boundaries. On the other hand, $\nu$ should have no such influence because of the instantaneous smoothing provided by convolution with the heat kernel. The first two results of this section make these observations precise. 
Theorem 6.1. Let $\mu$ and $\nu$ be as in Theorem 1.1 and assume additionally that $\mu$ has a density function $h$ which is continuous and positive on $\left(-b_{-}, b_{+}\right)^{c}$. Then the free boundaries $s_{ \pm}$provided by Theorem 1.1 are $C^{1}$ on the respective time intervals where they are finite.

Proof. Let $\left(u, s_{-}, s_{+}\right)$denote the solution of the given Stefan problem. For a given $\tau>0$ consider the (classical) Stefan problem with data $\tilde{b}_{ \pm}=s_{ \pm}(\tau)$, $\tilde{\varphi}(x)=u(x, \tau)$, and $\tilde{h}(x)=h(x) 1_{\left(-s_{-}(\tau), s_{+}(\tau)\right)^{c}}$. By Theorem A.4 of the appendix there exists $\delta>0$ and a triple $\left(\tilde{u}, \tilde{s}_{-}, \tilde{s}_{+}\right)$such that (A.5)-(A.8) hold for $0<t<\delta$. The functions $\tilde{s}_{ \pm}$are $C^{1}$ on $(0, \delta)$, provided they are finite. It is easy to deduce from Green's formula that the integral equations (1.7) for $\left(\tilde{u}, \tilde{s}_{-}, \tilde{s}_{+}\right)$then hold for $0<t<\delta$.

Now consider the Stefan problem (in the sense of Theorem 1.1) with data $b_{ \pm}^{*}=\tilde{s}_{ \pm}(\delta / 2), \quad \mu^{*}(d x)=h(x) 1_{\left(-\tilde{s}_{-}(\delta / 2), \tilde{s}_{+}(\delta / 2)\right)}(x) d x$, and $\nu^{*}(d x)=$ $\tilde{u}(x, \delta / 2) d x$. Let $\left(u^{*}, s_{-}^{*}, s_{+}^{*}\right)$ be the solution provided by Theorem 1.1.

Define $\bar{s}_{ \pm}$by $\bar{s}_{ \pm}(t)=\bar{s}_{ \pm}(t), 0<t \leq \tau, \bar{s}_{ \pm}(t)=\tilde{s}_{ \pm}(t-\tau), \tau<t \leq \tau+\delta / 2$, and $\bar{s}_{ \pm}(t)=s_{ \pm}^{*}(t-\tau-\delta / 2), \tau+\delta / 2<t<\infty$. Similarly, in the same respective ranges define $\bar{u}(x, t)=u(x, t), \tilde{u}(x, t-\tau)$, and $u^{*}(x, t-\tau-\delta / 2)$. Then it is easy to show that $\left(\bar{u}, \bar{s}_{-}, \bar{s}_{+}\right)$is a solution of the original Stefan problem. By Theorem 1.2 we conclude that $\left(\bar{u}, \bar{s}_{-}, \bar{s}_{+}\right)=\left(u, s_{-}, s_{+}\right)$. Since $\tau>0$ was arbitrary, it follows easily (see Remark A.5) that $s_{ \pm}$are $C^{1}$.

Theorem 6.2. Under the same hypotheses as Theorem 6.1 assume further that $h$ is $C^{k}\left(\right.$ resp. $\left.C^{\infty}\right)$ on some open interval I. Then $s_{ \pm}$are $C^{k+1}\left(\right.$ resp. $\left.C^{\infty}\right)$ on any time interval $J$ such that $s_{ \pm}(t) \in I$ for each $t$ in $J$.

Proof. Let $s_{+}(t) \in I$ for $\tau \leq t<\tau+\varepsilon$. Then $\left(u(x, t-\tau), s_{+}(t-\tau)\right)$ satisfies the (classical) one-sided Stefan problem on $\left\{(x, t): 0<x<s_{+}(t), 0<t<\varepsilon\right\}$ with initial data $\varphi(x)=u(x, \tau)$, latent heat $h$, and prescribed temperature on the fixed boundary $x=0$ given by $f(0, t)=u(0, t-\tau)$. One may then argue as in [21] (which considers the case $h \equiv 1$ ) with a few obvious changes.

We conclude this section by summarizing the information concerning the large time behavior of the free boundaries which emerged in the course of the proof of Theorem $1.2(\S 3)$.

Theorem 6.3. Let $\mu, \nu$, and $\left(u, s_{-}, s_{+}\right)$be as in Theorem 1.1. Let $(A, B)=$ $\left(-s_{-}(\infty), s_{+}(\infty)\right)$. Then $\|\mu\|<\|\nu\|$ implies $(A, B)=(-\infty, \infty)$. If $\|\mu\|>\|\nu\|$ then at least one of $A$ or $B$ is finite. Moreover, $(A, B)$ is the smallest open interval such that

$$
\mu((A, B))=\nu(\mathbb{R}),
$$

and

$$
\left|\int_{A}^{B} x \mu(d x)-\int x \nu(d x)\right| \text { is as small as possible. }
$$


If $\|\mu\|=\|\nu\|$ then $(A, B)$ is the smallest open interval with the following properties:

$$
\begin{gathered}
\text { support }(\mu) \subseteq(A, B), \\
A=-\infty \text { if } \int x \mu(d x)>\int x \nu(d x), \\
B=\infty \text { if } \int x \mu(d x)<\int x \nu(d x) .
\end{gathered}
$$

Proof. Only the statements concerning the case $\|\mu\|=\|\nu\|$ require proof since the remainder have been proved in $\S 3$. Also (6.3) has been proved in $\S 3$ so we may assume $\operatorname{support}(\mu)=(\alpha, \beta) \neq(-\infty, \infty)$. In particular, the integral $\int x \mu(d x)$ is well defined.

Suppose $\int x \mu(d x)>\int x \nu(d x)$ but $A>-\infty$. Then $\int_{-s_{-}(t)}^{s_{+}(t)} \mu(d x) \rightarrow \int \nu(d x)$, as explained in $\S 3$, and by Fatou's Lemma $\lim _{t \rightarrow \infty} \inf \int_{-s_{-}(t)}^{s_{+}(t)}(x-A) u(x, t) d x \geq$ 0 . Thus, the monotone convergence theorem and (1.7) yield $\int(x-A) \mu(d x) \leq$ $\int(x-A) \nu(d x)$. Hence, $\int x \mu(d x) \leq \int x \nu(d x)$, a contradiction. Thus, (6.4) holds. Statement $(6.5)$ is proved similarly.

Now the results of $\S 3$, in particular the identification of $\mu$ with the exit distribution of co-space-time Brownian motion, imply that $B$ may take only two values: $\beta$ and $\infty$. Moreover, if the latter holds and $\beta$ is finite, then $s_{+}(T)=\infty$ for some finite $T$. Analogous statements hold for $A$.

Now suppose $\int x \mu(d x)>\int x \nu(d x), \beta<\infty$, and $B=\infty$. Define a new measure $\mu^{\prime}$, having continuous distribution function, to agree with $\mu$ on $(-\infty, \beta)$ and have $\mu^{\prime}(\beta, \infty)>0$. Since $s_{+}(T)=\infty$ for some $T<\infty$, the solution of the new Stefan problem with data $\left(\nu, \mu^{\prime}\right)$ will agree with the old up to the smallest such $T$, but will differ thereafter due to the melting of the new mass on $(\beta, \infty)$. Moreover, if $\left(A^{\prime}, B^{\prime}\right)$ denote the new limits of the free boundaries, we can only have $\int_{A}^{B} x \mu^{\prime}(d x)>\int_{A}^{B} x \mu(d x)$, contradicting (6.2). Therefore $B=\beta$.

Similar considerations show that $A=\alpha$ in the case of (6.5), and that $(A, B)=(\alpha, \beta)$ if $\mu$ and $\nu$ have equal means. The proof is complete.

\section{OPEN QUESTIONS}

We list here some unsolved problems which, we believe, provide interesting directions for future research.

(I) Extend the results of this paper to higher dimensions and/or to the setting of parabolic equations more general than the heat equation. The Skorohod embedding theorem is known to fail for certain pairs $(\nu, \mu)$ in higher dimensions, so it may be necessary to impose further hypotheses on $\mu$ and $\nu$.

(II) Relax the hypotheses on $\mu$ and $\nu$ to $\mu \wedge \nu=0$. (P. Chacon [6] asserts that this is the only hypothesis needed for his result in the Brownian case.) In 
the absence of condition (1.5) the integral equations (1.7) must be modified to involve the gradient of $u$ along constant portions of the free boundaries.

(III) Give a direct proof of Theorem 6.1 without invoking the classical existence results (e.g., Theorem A.4).

(IV) If $\mu$ is only assumed absolutely continuous, must $s_{ \pm}$be absolutely continuous?

(V) Let us say that a positive (resp. negative) explosion occurs if $s_{+}(T)=\infty$ (resp. $s_{-}(T)=\infty$ ) for some finite $T$. Give necessary and sufficient conditions on the data for such explosions to occur.

(VI) Investigate in greater detail how the data influence the asymptotic behavior of $s_{ \pm}$as $t \rightarrow \infty$ and as $t \rightarrow 0$.

\section{APPENDIX}

We collect here for easy reference some results which may be proved by means of the classical methods presented in Chapter 17 of [5].

Theorem A.1. Let $b_{ \pm}$be given positive constants, $\varphi$ a nonnegative piecewise continuous function supported in $\left(-b_{-}, b_{+}\right)=I$, and $h=h_{0} \chi_{I} c$, where $h_{0}$ is a continuous function bounded below by $m>0$. There exists a triple $\left(u, s_{-}, s_{+}\right)$ such that $s_{ \pm}$are increasing $C^{1}$ functions on $(0, \infty)$, right continuous at 0 with $s_{ \pm}(0)=b_{ \pm}$, and $u$ is continuous on $\mathbf{R} \times(0, \infty)$. Moreover, the following hold:

$$
\begin{aligned}
& L u(x, t)=0, \quad-s_{-}(t)<x<s_{+}(t), 0<t<\infty, \\
& u(x, t)=0, \quad x \notin\left(-s_{-}(t), s_{+}(t)\right), 0<t<\infty,
\end{aligned}
$$

$$
u(x, t) \rightarrow \frac{1}{2}[\varphi(x+)+\varphi(x-)] \equiv \varphi_{a v}(x) \text { as } t \rightarrow 0,-b_{-}<x<b_{+},
$$

and,

$$
\frac{1}{2} u_{x}\left( \pm s_{ \pm}(t), t\right)=\mp h\left( \pm s_{ \pm}(t)\right) d s_{ \pm}(t) / d t, \quad 0<t<\infty .
$$

Theorem A.2 (Monotonicity). Suppose $\varphi^{(2)} \geq \varphi^{(1)}, h^{(2)} \leq h^{(1)}$, and $b_{ \pm}^{(2)} \geq b_{ \pm}^{(1)}$. Suppose $\left(u^{(i)}, s_{ \pm}^{(i)}\right)$ satisfy (A.1)-(A.4) for $i=1,2$, with $s_{ \pm}^{(i)}$ continuous on $[0, \infty)$ and $C^{1}$ on $(0, \infty)$. Then $s_{ \pm}^{(1)}(t) \leq s_{ \pm}^{(2)}(t)$ for all $t>0$.

Theorem A.3. Suppose $b_{ \pm}, \varphi$, and $h$ satisfy the same hypotheses as in Theorem A.1 except that we allow the support of $\varphi$ to be all of $\left[-b_{-}, b_{+}\right]$. Then the same conclusions hold as in Theorem A.1.

The proof uses the Monotonicity Theorem and is virtually identical to that of [5, Theorem 17.3.1].

As a further corollary of the Monotonicity Theorem we deduce that the solution $\left(u, s_{ \pm}\right)$of Theorem A.3 is unique.

Finally, we formulate a local version of Theorem A.3 . 
Theorem A.4. Let $b_{ \pm}$be given positive constants where we allow either one to take the value $\infty, \varphi$ a nonnegative bounded piecewise continuous function supported in $\left[-b_{-}, b_{+}\right]$, and $h$ a function which is continuous and positive on $\left[b_{+}, b_{+}+\varepsilon\right]$ and $\left[-b_{-}-\varepsilon, b_{-}\right]$for some $\varepsilon>0$. Then there exists a triple $\left(u, s_{-}, s_{+}\right)$and $\delta>0$ such that $s_{ \pm}$are nondecreasing extended real-valued continuous functions on $(0, \delta)$, right continuous at 0 with $s_{ \pm}(0)=b_{ \pm}$, and $u$ is continuous on $\mathbb{R} \times(0, \infty)$, such that

$$
\begin{gathered}
L u(x, t)=0, \quad-s_{-}(t)<x<s(t), 0<t<\delta, \\
u(x, t) \rightarrow 0, \quad \text { as } x \rightarrow \pm s_{ \pm}(t) \text { for each } 0<t<\delta, \\
u(x, t) \rightarrow \varphi_{a v}(x), \quad \text { as } t \rightarrow 0, \quad-b_{-}<x<b_{+},
\end{gathered}
$$

and

$$
\frac{1}{2} u_{x}\left( \pm s_{ \pm}(t), t\right)=\mp h\left( \pm s_{ \pm}(t)\right) d s / d t(t), \quad 0<t<\delta, \text { if } s_{ \pm}(t)<\infty .
$$

Remark A.5. The $\delta$ in Theorem A.4 may be chosen so as to depend only on $\inf (h)$, where $h$ denotes the extended function. This follows from the monotonicity theorem.

\section{REFERENCES}

1. J. Azema and M. Yor, Une solution simple au problème de Skorohod, Séminaire de Probabilités XIII, Lecture Notes in Math., vol. 721, Springer-Verlag, New York, 1979, pp. 90-115.

2. R. F. Bass, Skorohod stopping via stochastic integrals, Séminaire de Probabilités XVII, Lecture Notes in Math., vol. 986, Springer, New York, 1983, pp. 221-224.

3. J. Baxter and R. V. Chacon, Potentials of stopped distributions, Illinois J. Math. 18 (1974), 649-656.

4. L. Breiman, Probability, Addison-Wesley, Reading, Mass., 1968.

5. J. R. Canon, The one-dimensional heat equation, Encyclopedia of Mathematics and its Applications, Vol. 23, Addison-Wesley, Reading, Mass., 1984.

6. P. R. Chacon, The filling scheme and barrier stopping times, Ph. D. Dissertation, Univ. of Washington, Seattle, 1985.

7. R. Chacon and J. B. Walsh, One-dimensional potential embedding, Séminaire de Probabilités X, Lecture Notes in Math., vol. 511, Springer, New York, 1976, pp. 19-23.

8. J. L. Doob, Classical potential theory and its probabilistic counterpart, Springer-Verlag, Berlin, 1984.

9. L. Dubins, On a theorem of Skorohod, Ann. Math. Statist. 39 (1969), 2094-2097.

10. S. N. Ethier and T. G. Kurtz, Markov Processes: Characterization and convergence, Wiley, New York, 1986.

11. N. Falkner, On Skorohod embedding in n-dimensional Brownian motion by means of natural stopping times, Séminaire de Probabilités XIV, Lecture Notes in Math., vol. 784, Springer, New York, 1980, pp. 357-391.

12. D. Heath, Skorohod stopping via potential theory, Séminaire de Probabilités VIII, Lecture Notes in Math., vol. 381, Springer, New York, 1974, pp. 150-154.

13. K. Itô and H. P. McKean, Diffusion processes and their sample paths, Springer-Verlag, Berlin, 1974.

14. R. M. Loynes, Stopping times on Brownian motion: Some properties of Root's construction, Z. Wahrsch. Verw. Gebiete 16 (1970), 211-218. 
15. I. Meilijson, On the Azema-Yor stopping time, Séminaire de Probabilités XVII, Lecture Notes in Math., vol. 986, Springer, New York, 1983, pp. 225-226.

16. P. A. Meyer, Le schéma de remplissage en temps continu, Séminaire de Probabilités VI, Lecture Notes in Math., vol. 258, Springer, New York, 1972, pp. 130-150.

17. E. Perkins, The Cereteli-Davis solution of the $H^{1}$-embedding problem and an optimal embedding in Brownian motion, Seminar on Stochastic Processes (1985), Birkhäuser, Amsterdam, 1987.

18. D. H. Root, The existence of certain stopping times on Brownian motion, Ann. Math. Statist. 40 (1969), 715-718.

19. H. Rost, The stopping distributions of a Markov process, Invent. Math. 14 (1971), 1-16.

20. _ _ Skorohod stopping times of minimal variance, Séminarie de Probabilités X, Lecture Notes in Math., vol. 511, Springer, New York, 1976, pp. 194-208.

21. D. Schaeffer, A new proof of the infinite differentiability of the free boundary in the Stefan problem, J. Differential Equations 20 (1976), 266-269.

22. A. Schatz, Free boundary problems of Stefan type with prescribed flux, J. Math. Anal. Appl. 28 (1969), 569-579.

23. A. V. Skorohod, A limit theorem for sums of independent random variables, Dokl. Akad. Nauk SSSR 133 (1960), 34-35 (Russian).

24. __ Studies in the theory of random processes, Kiev Univ., 1961; Addison-Wesley, Reading, Mass. 1965 (translation).

25. P. Vallois, Le probleme de Skorohod sur $\mathbb{R}$ : Une approche avec le temps local, Séminaire de Probabilités XVIII, Lecture Notes in Math., vol. 986, Springer, New York, 1983, pp. 227-239.

26. P. van Moerbeke, On optimal stopping and free boundary problems, Arch. Rational Mech. Anal. 60 (1975), 101-148.

27. __ An optimal stopping problem with linear reward, Acta Math. 132 (1974), 111-151.

28. D. V. Widder, The heat equation, Academic Press, New York, 1975.

Department of Mathematics, Syracuse University, Syracuse, New York 13244-1150 